\title{
Adaptive tracking control for a class of uncertain switched stochastic nonlinear systems
}

\author{
Xikui Liu', Yanxin $\mathrm{Li}^{1}$ and Yan $\mathrm{Li}^{2 *}$ (i)
}

"Correspondence:

liyanhd@163.com

${ }^{2}$ College of Electrical Engineering

and Automation, Shandong

University of Science and

Technology, Qingdao, China

Full list of author information is

available at the end of the article

\begin{abstract}
This paper pursues an adaptive fuzzy control scheme for a class of nonlinear systems with stochastic switching. A general controller and adaptive mechanism are designed by utilizing Lyapunov function approach and backstepping technique. It is demonstrated that the presented control method can guarantee that all the signals in the closed-loop system are semi-globally uniformly ultimately bounded (SUUB) and the tracking error is convergent to a neighborhood of the origin. Finally, the simulation results verify the feasibility of the control strategy presented in this paper.
\end{abstract}

Keywords: Adaptive fuzzy control; Stochastic switched nonlinear systems; Backstepping technique

\section{Introduction}

As is well known, a common Lyapunov function (CLF) guarantees that the switched system is stable under arbitrary switching [1]. As an impactful approach for the stability analysis, CLF has been widely employed for control synthesis of switched linear systems [2-6]. For instance, [7] used the classic quadratic Lyapunov function and solved the stabilization problem for a class of stochastic nonlinear strict-feedback systems. Based on CLF method for a class of switched nonlinear systems, $[8,9]$ have investigated three state feedback control methods; however, the nonlinear functions of the above control systems are known. Additionally, the backstepping technique is used for the global stabilization problem for switched nonlinear systems in strict-feedback form under arbitrary switchings [8]. The adaptive backstepping approach is a recursive design methodology for controller design. It constructs associated Lyapunov functions and feedback control laws, and its main purpose is to design the adaptive laws and virtual control functions to counteract the unknown nonlinearity of system [10]. In recent years, in view of several classes of switched nonlinear systems, some backstepping control design methods have been proposed. Nevertheless, few of them take into account the uncertainties that exist extensively in practical switched nonlinear systems $[6,11]$.

In the last few decades, as a typical hybrid system, switched systems have been a great concern with their increasing significance in engineering practice, such as multiagent systems, aircraft-control systems and circuit and power systems [12,13]. Switched systems

(c) The Author(s) 2019. This article is distributed under the terms of the Creative Commons Attribution 4.0 International License (http://creativecommons.org/licenses/by/4.0/), which permits unrestricted use, distribution, and reproduction in any medium, provided you give appropriate credit to the original author(s) and the source, provide a link to the Creative Commons license, and indicate if changes were made. 
present switching between a set of subsystems resting with changing environmental factors. The system detects and breaks down various parameters in the changing environment, and then switches to the subsystem matching with the environment. So far, the controller design and stability analysis of switched systems have proposed remarkable results [2, 14-20]. In the actual control system, the dynamic characteristics of controlled objects such as production process, production equipment and transmission system are difficult to describe by accurate mathematical model. With the change of working environment, the components of the control system may be aged or damaged, and the characteristics of the controlled object also change. All these factors lead to some inevitable errors between the mathematical model of the controlled object and the actual object. For example, in large power systems, due to the large dimension, many systems contain unmodeled dynamic, uncertain parameters and random noise. In the actual operation, the system will also be affected by various harmonic and load disturbances. These random uncertainties of the power system bring about security risks to the normal operation of the power system. All of the above systems can be described by switching system. Since stochastic switched systems integrate the characteristics and difficulties of stochastic systems and switched systems, it is very difficult to analyze the stability and application of stochastic switched systems.

Obviously, stochastic disturbance is considered as one of the unstable sources of control systems which usually exists in many practical systems [21-23]. So, for a deterministic nonlinear system, the control for stochastic nonlinear system is much more difficult. Therefore, the research on control design and stability analysis for nonlinear stochastic systems is a significant and challenging subject, and it has been a topic of great concern in the last few years [24-28]. Specifically, some control methods based on adaptive backstepping technique for deterministic nonlinear systems [29-32] have been successfully generalized to nonlinear stochastic systems [33-39]. For instance, an output-feedback backstepping controller was developed for a class of stochastic nonlinear systems in [40], the state feedback controller is designed for nonlinear stochastic systems with Markovian switching [41] and [42] presented the backstepping control design approaches. Nevertheless, these methods are only suitable for those nonlinear stochastic systems with known nonlinear dynamic models. Adaptive output-feedback control methods for a class of uncertain nonlinear stochastic systems were proposed by utilizing the fuzzy logic system (FLS) and the stability of the control systems was discussed in [35]. The results of [35] were extended to a class of uncertain large-scale nonlinear stochastic systems. The approaches $[17,20]$ decreased the adjustable parameters. The presented controller in [43] has a simple structure because the unknown virtual control signals were directly approximated via FLS. From the above, adaptive fuzzy control approach plays an important role in dealing with uncertain nonlinear systems.

Motivated by the above discussions, this paper presents an adaptive fuzzy tracking control method for a class of uncertain strict-feedback switched nonlinear systems with completely unknown nonlinear functions. In the design process, the unknown nonlinear functions are approximated by utilizing FLS, an adaptive fuzzy control has been proposed by the backstepping technique and CLF method. Compared with the previous results, the advantages of this paper can be summarized as follows.

1. This paper studies the tracking control problem of switched nonlinear uncertain systems, which is different from the available methods on switched nonlinear 
systems. The stochastic disturbance is considered and all system functions studied in this paper are unknown completely. Therefore, compared with existing work, the controlled system is more general and the control design is more challenging.

2. There are two kinds of adaptive fuzzy backstepping control approaches proposed in this paper for a class of switched nonlinear uncertain systems. We propose a design approach with multiple adaptive laws in the first place. After that, another approach with only one adaptive law is presented in order to avoid too many parameters. In addition, we use the norm of the unknown weight vector of FLS basis function rather than the weight vector elements themselves as the estimated parameter at each step, which significantly reduces the number of adaptive parameters. Therefore, the presented control design approach becomes more practical to use.

The remainder of manuscript is organized as follows. The preliminaries and problem formulation are addressed in Sect. 2. A novel adaptive fuzzy control scheme is introduced in Sect. 3. A simulation example is developed in Sect. 4, Finally, conclusions are given in Sect. 5.

\section{Preliminaries and problem formulation}

The following notations are used in this paper. $R_{+}$means the set of all non-negative real numbers, $R^{n}$ represents the real $n$-dimensional space, and $R^{n \times r}$ stands for the set of all $n \times r$ real matrices. $\|X\|$ indicates the Euclidean norm of a vector $x . C^{2,1}$ represents the set of all the functions $V(x, t)$ which belong to $C^{2}$ with respect to $x$ and belong to $C^{1}$ with respect to $t$. $\operatorname{Tr}(A)$ means a trace of the matrix $A$.

\subsection{Stochastic stability}

Consider a stochastic nonlinear system of the following form:

$$
d x=f(x, t) d t+g(x, t) d \omega
$$

where $x \in R^{n}$ is the state variable, $f: R^{n} \times R^{+} \rightarrow R^{n}, h: R^{n} \times R^{+} \rightarrow R^{n \times r}$ are continuous functions, and $\omega$ represents an independent $r$-dimension standard Brownian motion defined on the complete probability space $\left(\Omega, F,\left\{F_{t}\right\}_{t \geq 0}, P\right)$ with $\Omega$ representing a sample space, and $F$ being a sample $\sigma$-field, $\left\{F_{t}\right\}_{t \geq 0}$ representing a filtration and $P$ representing a probability measure.

Definition 1 ([44]) For the twice continuously differentiable function $\mathcal{V}(x, t)$, the differential operator $L$ is defined as:

$$
L \mathcal{V}=\frac{\partial \mathcal{V}}{\partial x} f+\frac{\partial \mathcal{V}}{\partial t}+\frac{1}{2} \operatorname{Tr}\left\{h^{T} \frac{\partial^{2} \mathcal{V}}{\partial x^{2}} h\right\}
$$

Remark 1 The term $\frac{1}{2} \operatorname{Tr}\left\{h^{T} \frac{\partial^{2} \mathcal{V}}{\partial x^{2}} h\right\}$ is called Itô correction term, $\frac{\partial^{2} \mathcal{V}}{\partial x^{2}}$ will be more difficult to construct the common virtual control function and the unified adaptive mechanism for uncertain switched stochastic systems than that of deterministic system.

Lemma 1 ([45]) Consider the dynamic system as follows:

$$
\dot{\hat{\zeta}}=-\gamma \hat{\zeta}(t)+\chi \rho(t)
$$


where $\gamma>0, \chi>0$ and $\rho(t)>0$ is a function, then, for bounded initial condition $\forall \hat{\zeta}\left(t_{0}\right) \geq 0$, $\varsigma(t) \geq 0$ for $\forall t \geq t_{0}$.

Lemma 2 ([34]) Suppose there is a function $\mathcal{V}(x, t) \in C^{2,1}$, and constants $p>0$ and $q>0$, class $k_{\infty}$ functions $\bar{\alpha}_{1}$ and $\bar{\alpha}_{2}$, such that

$$
\left\{\begin{array}{l}
\bar{\alpha}_{1}(\|x\|) \leq \mathcal{V}(x, t) \leq \bar{\alpha}_{2}(\|x\|) \\
L \mathcal{V} \leq-p V(x, t)+q
\end{array}\right.
$$

for $\forall x \in R^{n}$ and $\forall t>0$. Then there exists an unique strong solution of system (1) for each $x_{0} \in R^{n}$ and the system is bounded in probability.

Lemma 3 (Young's inequality [46]) For $\forall(x, y) \in R^{2}$, there is an inequality as follows:

$$
x y \leq \frac{\varepsilon^{m}}{m}|x|^{m}+\frac{1}{n \varepsilon^{n}}|y|^{n},
$$

where $\varepsilon>0, m>1, n>1$ and $(m-1)(n-1)=1$.

\subsection{Problem formulation}

Consider a class of switched nonlinear system in the following form:

$$
\begin{aligned}
d x_{j} & =\left[h_{j, \tau(t)} x_{j+1}+f_{j, \tau(t)}\left(\bar{x}_{j}\right)\right] d t+\psi_{n, \tau(t)}^{T}\left(\bar{x}_{j}\right) d \omega, \\
j & =\{1,2, \ldots, n-1\} \in I, \\
d x_{n} & =\left[h_{n, \tau(t)} u_{\tau(t)}+f_{n, \tau(t)}\left(\bar{x}_{n}\right)\right] d t+\psi_{n, \tau(t)}^{T}\left(\bar{x}_{n}\right) d \omega, \\
y & =x_{1},
\end{aligned}
$$

where $\bar{x}_{j}=\left(x_{1}, x_{2}, \ldots, x_{j}\right)^{T} \in R^{j}, j=1,2, \ldots, n$ is the system state, $\tau(t)=k(k \in M)$ implies that the $k$ th subsystem is active, $\omega$ is defined in (1), $y$ is the system output; $\tau(t):[0,+\infty) \rightarrow$ $M=\{1,2, \ldots, m\}$ is the switching signal; $u_{r} \in R$ denotes the control input of the $r$ th subsystem. For any $j=1,2, \ldots, n$ and $r=1,2, \ldots, m, f_{j, r}\left(\bar{x}_{j}\right)$ is an unknown smooth nonlinear function being the system uncertainty, and $h_{j, r}>0$ is a constant.

Assumption 1 ([47]) The tracking target $y_{v}(t)$ and its time derivatives up to the $n$th order are continuous and bounded.

Remark 2 When we do not consider the unknown functions and the tracking control problem, system (3) will be reduced to system (1) in [17], So, the system studied in this note is more general.

Assumption 2 ([48]) For $j \in I$, there exist unknown constants $b_{k}$ and $b_{K}$ such that

$$
0<b_{k} \leq\left|h_{j, r} x_{j+1}\right| \leq b_{K}<\infty, \quad \forall x_{j+1} \in R^{j} \times R
$$

In addition, the sign of $h_{j, r} x_{j+1}$ is known, and the sign of $h_{n, r} u_{\sigma(t)}$ is unknown. Without loss of generality, it is further assumed that $h_{j, r} x_{j+1} \geq b_{k}>0$. 
Remark 3 In the existing researches on pure-feedback nonlinear systems, it is usually considered that the sign of $h_{n, r} u_{\tau(t)}$ is known. Therefore, Assumption 2 is reasonable, it is a meaningful work for the stochastic nonlinear systems.

\subsection{Fuzzy logic systems}

In the process of controller design and stability analysis, the FLS is adopted in order to approximate the unknown functions.

Consider the $j$ th IF-THEN rule of the following form:

$R_{j}$ : IF $\bar{x}_{1}$ is $\Gamma_{1}^{j}$ and $\ldots$ and $\bar{x}_{n}$ is $\Gamma_{n}^{j}$, then $y$ is $P^{j}, j=1,2, \ldots, \aleph$,

where $x=\left[\bar{x}_{1}, \bar{x}_{2}, \ldots, \bar{x}_{n}\right]^{T} \in R^{n}$, and $y \in R$ are input and output of the FLS, respectively. $\Gamma_{1}^{j}, F_{2}^{j}, \ldots, \Gamma_{n}^{j}$ and $P^{j}$ are fuzzy sets in $R$. By using the singleton fuzzification, the product inference and the center-average defuzzification, the fuzzy logic system can be expressed as

$$
y(\bar{x})=\frac{\sum_{j=1}^{\aleph} \iota_{j} \prod_{i=1}^{n} \mu_{\Gamma_{i}^{j}}\left(\bar{x}_{i}\right)}{\sum_{j=1}^{\aleph}\left[\prod_{i=1}^{n} \mu_{\Gamma_{i}^{j}}\left(\bar{x}_{i}\right)\right]},
$$

where $\aleph$ is the number of IF-THEN rules, $\varpi_{j}$ is the point at which fuzzy membership function $\mu_{P^{j}}\left(\varpi_{j}\right)=1$. Let

$$
\zeta_{l}(\bar{x})=\frac{\prod_{i=1}^{n} \mu_{\Gamma_{i}^{j}}\left(\bar{x}_{i}\right)}{\sum_{j=1}^{\aleph}\left[\prod_{i=1}^{n} \mu_{\Gamma_{i}^{j}}\left(x_{i}\right)\right]},
$$

where $\zeta(\bar{x})=\left[\zeta_{1}(\bar{x}), \zeta_{2}(\bar{x}), \ldots, \zeta_{\aleph}(\bar{x})\right]^{T}$ and $\Im=\left[\varphi_{1}, \varphi_{2}, \ldots, \varphi_{\aleph}\right]^{T}, \varphi_{j}=\max _{y \in R} \mu_{P}^{j}(y)$. Then the fuzzy logic system can be described as

$$
y=\varphi^{T} \zeta(\bar{x})
$$

Lemma 4 ([49]) Let $f(\bar{x})$ be a continuous function defined on a compact set $\Omega$. Then, for $\forall \epsilon>0$, there exists a fuzzy logic system (4) such that

$$
\sup _{\bar{x} \in \Omega}\left|f(\bar{x})-\Im^{T} \zeta(\bar{x})\right| \leq \epsilon .
$$

Remark 4 Lemma 4 shows that real continuous function $f(\bar{x})$ can be expressed as a linear combination of bounded error $\epsilon$-based function vectors $\zeta(\bar{x})$. That is, $f(\bar{x})=\Im_{\Im}^{T} \zeta(\bar{x})+\xi(\epsilon)$, $|\xi(\epsilon)|<\epsilon$, it plays an important role in the whole process of adaptive laws design. It is noted that $0<\zeta^{T} \zeta \leq 1$.

\section{Main results}

In this section, the adaptive fuzzy control scheme of system (3) is proposed by combining the FLS with adaptive backstepping technique and CLF approach. In Sect. 3.1, a specific design process will be given. In each step, we will design a virtual control function $\sigma_{i}$ via using a proper CLF $V_{i}$, and the control law $u_{k}$ will finally be designed. In Sect. 3.2, in order to avoid repetition, a final CLF will be only adopted to prove the design procedure. 


\subsection{Adaptive control design under multiple adaptive laws}

In this section, a multiple adaptive control method based on backstepping technique is presented for the system (3). The backstepping design procedure contains $n$ steps and it is developed via the following coordinate transformation:

$$
z_{1}=x_{1}-y_{v}, \quad z_{i}=x_{i}-\sigma_{j-1}, \quad j=1,2, \ldots, n,
$$

where $\sigma_{j-1}$ is considered an intermediate control function.

Define: $\bar{y}_{v}^{(t)}=\left[y_{v}, y_{v}^{(1)}, \ldots, y_{v}^{(j)}\right]^{T}, j=1,2, \ldots, n$, with $y_{v}^{(j)}$ denoting the $j$ th derivative of $y_{v}$.

At step $j$ of the design process, the unknown function $\hat{f}_{j, r}$ is approximated by a FLS $\Im_{j, r}\left(x_{j}\right)$. For this purpose, define a constant $\varsigma_{j}=\frac{\left\|\Im_{j, r}\right\|^{2}}{b_{k}}, j=1,2, \ldots, n$, denote $\hat{\zeta}_{j}$ as the estimation of $\varsigma_{j}$, and the estimation error is $\tilde{\zeta}_{j}=\varsigma_{j}-\hat{\zeta}_{j}$.

Now, we give detailed backstepping design process in the following steps.

Step 1. For stochastic pure-feedback system (3), according to the tracking error $z_{1}=$ $x_{1}-y_{v}$, the error dynamic is

$$
d z_{1}=\left[h_{1, r} x_{2}+f_{1, r}\left(\bar{x}_{1}\right)-\dot{y}_{v}\right] d t+\psi_{1, r}^{T}\left(\bar{x}_{1}\right) d \omega .
$$

To stabilize the subsystem (6), we choose such a stochastic Lyapunov function candidate defined by

$$
V_{1}=\frac{1}{4} z_{1}^{4}+\frac{b_{k}}{2 \ell_{1}} \tilde{\varsigma}_{1}^{2},
$$

where $\ell_{1}>0$ is a design constant. By (2), (5) and (6), one has

$$
\begin{aligned}
L V_{1}= & z_{1}^{3}\left[h_{1, r}\left(z_{2}+\sigma_{1}\right)+f_{1, r}\left(\bar{x}_{1}\right)-\dot{y}_{v}\right] \\
& +\frac{3}{2} z_{1}^{2} \psi_{1, r}^{T} \psi_{1, r}-\frac{b_{k}}{\ell_{1}} \tilde{\zeta}_{1} \dot{\hat{\zeta}}_{1} .
\end{aligned}
$$

From Lemma 3 and Assumption 2, the following inequalities hold:

$$
\begin{aligned}
& \frac{3}{2} z_{1}^{2} \psi_{1, r}^{T} \psi_{1, r} \leq \frac{3}{4} l_{1}^{-2} z_{1}^{4}\left\|\psi_{1, r}\right\|^{4}+\frac{3}{4} l_{1}^{2}, \\
& h_{1, r} z_{1}^{3} z_{2} \leq \frac{3}{4} b_{K} z_{1}^{4}+\frac{b_{K}}{4} z_{2}^{4},
\end{aligned}
$$

where $l_{1}>0$ is a design constant. Then substituting (8) and (9) into (7) yields

$$
\begin{aligned}
L V_{1} \leq & z_{1}^{3}\left[f_{1, r}+\frac{3}{4} b_{K} z_{1}+h_{1, r} \sigma_{1}-\dot{y}_{v}+\frac{3}{4} l_{1}^{2}\right. \\
& \left.+\frac{3}{4} l_{1}^{-2} z_{1}\left\|\psi_{1, r}\right\|^{4}\right]+\frac{b_{K}}{4} z_{2}^{4}-\frac{b_{k}}{\ell_{1}} \tilde{\varsigma}_{1} \dot{\hat{\zeta}}_{1} .
\end{aligned}
$$

Define $\hat{f}_{1, r}=f_{1, r}+\frac{3}{4} b_{K} z_{1}-\dot{y}_{v}+\frac{3}{4} l_{1}^{-2} z_{1}\left\|\psi_{1, r}\right\|^{4}+\left(k_{1}+\frac{3}{4}\right) z_{1}$, where $k_{1}>0$ is a design constant. Then the inequality (10) can be constructed as

$$
\begin{aligned}
L V_{1} \leq & -k_{1} z_{1}^{4}+z_{1}^{3} h_{1, r} \sigma_{1}+z_{1}^{3} \hat{f}_{1, r}-\frac{3}{4} z_{1}^{4}+\frac{3}{4} l_{1}^{2} \\
& +\frac{b_{K}}{4} z_{2}^{4}-\frac{b_{k}}{\ell_{1}} \tilde{\zeta}_{1} \dot{\hat{\zeta}}_{1} .
\end{aligned}
$$


$\hat{f}_{1, r}$ contains the unknown function $f_{1, r}$ and $\varphi_{1}$. According to Lemma 4 , for $\forall \epsilon_{1, r}>0$, there exists a FLS $\Im_{1, r}^{T} \zeta_{1, r}\left(\bar{X}_{1}\right)$ such that

$$
\hat{f}_{1, r}=\Im_{1, r}^{T} \zeta_{1, r}\left(\bar{X}_{1}\right)+\xi_{1, r}\left(\bar{X}_{1}\right),\left|\xi_{1, r}\left(\bar{X}_{1}\right)\right| \leq \epsilon_{1, r}
$$

where $\bar{X}_{1}=\left(x_{1}, y_{v}, \dot{y}_{v}\right)$.

Remark 5 Note that the FLS is directly used to approximate unknown nonlinear function $\hat{f}_{1, r}$ rather than only the unknown function $f_{1, r}$. This method will be used in the remaining design steps.

In the method of Young's inequality, it follows that

$$
\begin{aligned}
z_{1}^{3} \hat{f}_{1, r} & =z_{1}^{3} \frac{\Im_{1, r}^{T}}{\left\|\Im_{1, r}\right\|}\left\|\Im_{1, r}\right\| \zeta_{1, r}-z_{1}^{3} \xi_{1, r} \\
& \leq \frac{b_{k} z_{1}^{6}}{2 a_{1, r}^{2}} \frac{\left\|\Im_{1, r}\right\|^{2}}{b_{k}} \zeta_{1, r}^{T} \zeta_{1, r}+\frac{1}{2} a_{1, r}^{2}+\frac{3}{4} z_{1}^{4}+\frac{1}{4} \epsilon_{1, r}^{4} \\
& =\frac{b_{k}}{2 a_{1, r}^{2}} z_{1}^{6} \zeta_{1} \zeta_{1, r}^{T} \zeta_{1, r}+\frac{1}{2} a_{1, r}^{2}+\frac{3}{4} z_{1}^{4}+\frac{1}{4} \epsilon_{1, r}^{4},
\end{aligned}
$$

where $a_{1, r}$ is a design parameter and $\varsigma_{1}=\frac{\left\|\widetilde{\Im}_{1, r}\right\|^{2}}{b_{k}}$ is an unknown constant.

Let us take the virtual control signal as

$$
\sigma_{1}=-k_{1} z_{1}-\frac{1}{2 a_{1, \min }^{2}} \hat{\zeta_{1}} z_{1}^{3} \zeta_{1, r}^{T} \zeta_{1, r}
$$

The adaptation law is defined as

$$
\dot{\hat{\zeta}}_{1}=\frac{\ell_{1}}{2 a_{1, r}^{2}} z_{1}^{6} \zeta_{1, r}^{T} \zeta_{1, r}-\gamma_{1} \hat{\zeta}_{1}, \quad \hat{\zeta}_{1}(0) \geq 0,
$$

where $\gamma_{1}>0$ is a design constant.

By Assumption 2, Lemma 1, and the virtual control signal (13), we obtain

$$
z_{1}^{3} h_{1, r} \sigma_{1} \leq-k_{1} b_{k} z_{1}^{4}-\frac{b_{k}}{2 a_{1, r}^{2}} z_{1}^{6} \hat{\varsigma}_{1} \zeta_{1, r}^{T} \zeta_{1, r}
$$

Substituting (12), (13), (14), (15) into (11), we have

$$
\begin{aligned}
L V_{1} \leq & -k_{1}\left(1+b_{k}\right) z_{1}^{4}+\frac{b_{K}}{4} z_{2}^{4}+\frac{3}{4} l_{1}^{2}+\frac{1}{4} \epsilon_{1, r}^{2} \\
& +\frac{b_{k} \gamma_{1}}{\ell_{1}} \tilde{\zeta}_{1} \hat{\zeta}_{1}+\frac{1}{2} a_{1, r}^{2} .
\end{aligned}
$$

It is noted that

$$
\frac{b_{k} \gamma_{1}}{\ell_{1}} \tilde{\zeta}_{1} \hat{\zeta}_{1} \leq-\frac{b_{k} \gamma_{1}}{2 \ell_{1}} \tilde{\varsigma}_{1}^{2}+\frac{b_{k} \gamma_{1}}{2 \ell_{1}} \varsigma_{1}^{2}
$$


Combining (16) with (17) gives

$$
L V_{1} \leq-c_{1} z_{1}^{4}-\frac{b_{k} \gamma_{1}}{2 \ell_{1}} \tilde{\varsigma}_{1}^{2}+\varrho_{1}+\frac{b_{K}}{4} z_{2}^{4}
$$

where $c_{1}=k_{1}\left(1+b_{k}\right)$ and $\varrho_{1}=\frac{3}{4} l_{1}^{2}+\frac{1}{2} a_{1, r}^{2}+\frac{1}{4} \epsilon_{1, r}^{4}+\frac{b_{k} \gamma_{1}}{2 \ell_{1}} \varsigma_{1}^{2}$.

Step 2. Define $z_{2}=x_{2}-\sigma_{1}$ and with the Itô formula one has

$$
\begin{aligned}
d z_{2}= & {\left[h_{2, r}\left(x_{3}\right)+f_{2, r}\left(\bar{x}_{2}\right)-L \sigma_{1}\right] d t } \\
& +\left(\psi_{2, r}-\frac{\partial \sigma_{1}}{\partial x_{1}} \psi_{2, r}\right)^{T} d \omega,
\end{aligned}
$$

with

$$
\begin{aligned}
L \sigma_{1}= & \frac{\partial \sigma_{1}}{\partial x_{1}}\left[h_{1, r}\left(x_{2}\right)+f_{1, r}\left(\bar{x}_{2}\right)\right]+\frac{\partial \sigma_{2}}{\partial \hat{\zeta}_{1}} \dot{\hat{\zeta}}_{1} \\
& +\sum_{j=0}^{1} \frac{\partial \sigma_{1}}{\partial y_{v}^{(j)}} y_{v}^{(j+1)}+\frac{1}{2} \frac{\partial^{2} \sigma_{1}}{\partial x_{1}^{2}} \psi_{1, r}^{T} \psi_{1, r} .
\end{aligned}
$$

Consider the stochastic Lyapunov function as follows:

$$
V_{2}=V_{1}+\frac{1}{4} z_{2}^{4}+\frac{b_{k}}{2 \ell_{2}} \tilde{\zeta}_{2}^{2},
$$

where $\ell_{2}>0$ is a design constant. Similar to the analysis of (7), the following result holds:

$$
\begin{aligned}
L V_{2}= & L V_{1}+z_{2}^{3}\left[h_{2, r}\left(z_{3}+\sigma_{2}\right)+f_{2, r}\left(\bar{x}_{2}\right)-L \sigma_{1}\right] \\
& +\frac{3}{2} z_{2}^{2}\left(\psi_{2, r}-\frac{\partial \sigma_{1}}{\partial x_{1}} \psi_{1, r}\right)^{T}\left(\psi_{2, r}-\frac{\partial \sigma_{1}}{\partial x_{1}} \psi_{1, r}\right) \\
& -\frac{b_{k}}{\ell_{2}} \tilde{\zeta}_{2} \dot{\hat{\zeta}}_{2} .
\end{aligned}
$$

Note that

$$
\begin{aligned}
\frac{3}{2} z_{2}^{2}\left\|\psi_{2, r}-\frac{\partial \sigma_{1}}{\partial x_{1}} \psi_{1, r}\right\|^{2} \leq & \frac{3}{4} l_{2}^{-2} z_{2}^{4}\left\|\psi_{2, r}-\frac{\partial \sigma_{1}}{\partial x_{1}} \psi_{1, r}\right\|^{4} \\
& +\frac{3}{4} l_{2}^{2} \\
h_{2, r} z_{2}^{3} z_{3} \leq & \frac{3}{4} b_{K} z_{2}^{4}+\frac{b_{K}}{4} z_{3}^{4},
\end{aligned}
$$

where $l_{2}>0$ is a design constant. Substituting (18), (20) and (21) into (19), one derives

$$
\begin{aligned}
L V_{2} \leq & -c_{1} z_{1}^{4}-\frac{b_{k} \gamma_{1}}{2 \ell_{1}} \tilde{\varsigma}_{1}^{2}+\varrho_{1}+\frac{3}{4} l_{2}^{2}+\frac{b_{K}}{4} z_{3}^{4}-\frac{b_{k}}{\ell_{2}} \tilde{\zeta}_{2} \dot{\hat{\zeta}}_{2} \\
& +z_{2}^{3}\left[h_{2, r} \sigma_{2}+f_{2, r}\left(\bar{x}_{2}\right)+\frac{3}{4} b_{K} z_{2}+\frac{1}{4} b_{k} z_{2}\right. \\
& \left.-L \sigma_{1}+\frac{3}{4} l_{2}^{-2} z_{2}\left\|\psi_{2, r}-\frac{\partial \sigma_{1}}{\partial x_{1}} \psi_{1, r}\right\|^{4}\right] .
\end{aligned}
$$


Set

$$
\begin{aligned}
\hat{f}_{2, r}= & f_{2, r}-L \sigma_{1}+\frac{3}{4} l_{2}^{-2} z_{2}\left\|\psi_{2, r}-\frac{\partial \sigma_{1}}{\partial x_{1}} \psi_{1, r}\right\|^{4} \\
& +b_{K} z_{2}+\left(k_{2}+\frac{3}{4}\right) z_{2}
\end{aligned}
$$

with $k_{2}>0$ being a design parameter. Furthermore, (22) can be rewritten as

$$
\begin{aligned}
L V_{2} \leq & -c_{1} z_{1}^{4}-\frac{b_{k} \gamma_{1}}{2 \ell_{1}} \tilde{\varsigma}_{1}^{2}+\varrho_{1}+z_{2}^{3} h_{2, r} \sigma_{2}-k_{2} z_{2}^{4}+\frac{3}{4} l_{2}^{2} \\
& +z_{2}^{3} \hat{f}_{2, r}-\frac{3}{4} z_{2}^{4}+\frac{b_{K}}{4} z_{3}^{4}-\frac{b_{k}}{\ell_{2}} \tilde{\zeta}_{2} \dot{\hat{\zeta}}_{2} .
\end{aligned}
$$

$\hat{f}_{2, r}$ contains the unknown function $f_{1, r}, \psi_{1, r}$ and $\psi_{2, r}, \hat{f}_{2, r}$ cannot be realized in practical applications. The FLS $\Im_{2, r}^{T} \zeta_{2, r}\left(\bar{X}_{2}\right)$ is thus used to approximate $\hat{f}_{2, r}$, where $\bar{X}_{2}=$ $\left[\bar{x}_{2}^{T}, \hat{\zeta}_{1}, \bar{y}_{v}^{(2) T}\right]^{T} \in \Omega_{z_{2}} \subset R^{6}$. According to Lemma $4, \hat{f}_{2, r}$ can be described as

$$
\hat{f}_{2, r}=\mathfrak{\Im}_{2, r}^{T} \zeta_{2, r}\left(\bar{X}_{2}\right)+\xi_{2, r}\left(\bar{X}_{2}\right),\left|\xi_{2, r}\left(\bar{X}_{2}\right)\right| \leq \epsilon_{2, r}
$$

where $\forall \epsilon_{2, r}>0$ is a constant. In addition, using the same method used in (12) yields

$$
z_{2}^{3} \hat{f}_{2, r} \leq \frac{b_{k}}{2 a_{2, r}^{2}} z_{2}^{6} \varsigma_{2} \zeta_{2, r}^{T} \zeta_{2, r}+\frac{1}{2} a_{2, r}^{2}+\frac{3}{4} z_{2}^{4}+\frac{1}{4} \epsilon_{2, r}^{4}
$$

where $a_{2, r}>0$ is a design parameter and $\varsigma_{2}=\frac{\left\|\widetilde{I}_{2}, r\right\|^{2}}{b_{k}}$ is an unknown constant.

The virtual control signal is given by

$$
\sigma_{2}=-k_{2} z_{2}-\frac{1}{2 a_{2, \min }^{2}} \hat{\zeta}_{2} z_{2}^{3} \zeta_{2, r}^{T} \zeta_{2, r}
$$

Define the adaptive law as follows:

$$
\dot{\hat{\zeta}}_{2}=\frac{\ell_{2}}{2 a_{2, r}^{2}} z_{2}^{6} \zeta_{2, r}^{T} \zeta_{2, r}-\gamma_{2} \hat{\zeta}_{2}, \quad \hat{\zeta}_{2}(0) \geq 0,
$$

where $\gamma_{2}>0$ is a design constant.

Similar to (15) and (17), it is easy to obtain

$$
\begin{aligned}
& z_{2}^{3} h_{2, r} \sigma \leq-k_{2} b_{k} z_{2}^{4}-\frac{b_{k}}{2 a_{2, r}^{2}} z_{2}^{6} \hat{\varsigma}_{2} \zeta_{2, r}^{T} \zeta_{2, r}, \\
& \frac{b_{k} \gamma_{2}}{\ell_{2}} \tilde{\zeta}_{2} \hat{\zeta}_{2} \leq-\frac{b_{k} \gamma_{2}}{2 \ell_{2}} \tilde{\zeta}_{2}^{2}+\frac{b_{k} \gamma_{2}}{2 \ell_{2}} \varsigma_{2}^{2} .
\end{aligned}
$$

Substituting (24), (25), (26), (27), (28) into (23) results in

$$
L V_{2} \leq-\sum_{i=1}^{2}\left(c_{i} z_{i}^{4}+\frac{b_{k} \gamma_{i}}{2 \ell_{i}} \tilde{s}_{i}^{2}\right)+\sum_{i=1}^{2} \varrho_{i}+\frac{b_{K}}{4} z_{3}^{4},
$$

where $c_{i}=k_{i}\left(1+b_{k}\right)$ and $\varrho_{i}=\frac{3}{4} l_{i}^{2}+\frac{1}{2} a_{i, r}^{2}+\frac{1}{4} \epsilon_{i, r}^{4}+\frac{b_{k} \gamma_{i}}{2 \ell_{i}} \varsigma_{i}^{2}, i=1,2$. 
Step $j$. $(3 \leq j \leq n-1)$ Considering $z_{j}=x_{j}-\sigma_{j-1}$ and Itô's formula, we have

$$
\begin{aligned}
d z_{j}= & \left(h_{j, r}\left(x_{j+1}\right)+f_{j, r}\left(\bar{x}_{j}\right)-L \sigma_{j-1}\right) d t \\
& +\left(\psi_{j, r}-\sum_{i=1}^{j-1} \frac{\partial \sigma_{j-1}}{\partial x_{i}} \psi_{i, r}\right)^{T} d \omega,
\end{aligned}
$$

where

$$
\begin{aligned}
L \sigma_{j-1}= & \sum_{i=1}^{j-1} \frac{\partial \sigma_{j-1}}{\partial x_{i}}\left[h_{i, r}\left(x_{i+1}\right)+f_{i, r}\left(\bar{x}_{i}\right)\right] \\
& +\sum_{i=1}^{j-1} \frac{\partial \sigma_{j-1}}{\partial \hat{\zeta}_{i}} \dot{\hat{\zeta}}_{i}+\sum_{i=0}^{j-1} \frac{\partial \sigma_{j-1}}{\partial y_{v}^{(i)}} y_{v}^{(i+1)} \\
& +\frac{1}{2} \sum_{p, q=1}^{j-1} \frac{\partial^{2} \sigma_{j-1}}{\partial x_{p} \partial x_{q}} \psi_{p, r}^{T} \psi_{q, r} .
\end{aligned}
$$

The Lyapunov function is constructed in the following form:

$$
V_{j}=V_{j-1}+\frac{1}{4} z_{j}^{4}+\frac{b_{k}}{2 \ell_{j}} \tilde{\zeta}_{j}^{2},
$$

where $\ell_{j}>0$ is a design constant.

By following the same process used in Step 1, it follows that

$$
\begin{aligned}
L V_{j}= & L V_{j-1}+z_{j}^{3}\left[h_{j, r}\left(z_{j+1}+\sigma_{j}\right)+f_{j, r}\left(\bar{x}_{j}\right)-L \sigma_{j-1}\right] \\
& -\frac{b_{k}}{\ell_{j}} \tilde{\zeta}_{j} \dot{\hat{\zeta}}_{j}+\frac{3}{2} z_{j}^{2}\left(\psi_{j, r}-\sum_{i=1}^{j-1} \frac{\partial \sigma_{j-1}}{\partial x_{i}} \psi_{i, r}\right)^{T}\left(\psi_{j, r}\right. \\
& \left.-\sum_{i=1}^{j-1} \frac{\partial \sigma_{j-1}}{\partial x_{i}} \psi_{i, r}\right) .
\end{aligned}
$$

From the completion of squares and Lemma 3, the following inequalities hold:

$$
\begin{aligned}
\frac{3}{2} z_{j}^{2}\left\|\psi_{j, r}-\sum_{i=1}^{j-1} \frac{\partial \sigma_{j-1}}{\partial x_{i}} \psi_{i, r}\right\|^{2} \leq & \frac{3}{4} l_{j}^{2} \\
& +\frac{3}{4} l_{j}^{-2} z_{j}^{4}\left\|\psi_{j, r}-\sum_{i=1}^{j-1} \frac{\partial \sigma_{j-1}}{\partial x_{i}} \psi_{i, r}\right\|^{4}, \\
h_{j, r} z_{j}^{3} z_{j+1} \leq \frac{3}{4} b_{K} z_{j}^{4}+\frac{b_{K}}{4} z_{j+1}^{4}, &
\end{aligned}
$$

where $l_{j}$ is a design constant. Combining the above inequalities with (29) gives

$$
\begin{aligned}
L V_{j} \leq & -\sum_{i=1}^{j-1}\left(c_{i} z_{i}^{4}+\frac{b_{k} \gamma_{i}}{2 \ell_{1}} \tilde{\zeta}_{i}^{2}\right)+\sum_{i=1}^{j-1} \varrho_{i}+\frac{3}{4} l_{j}^{2}-k_{j} z_{j}^{4} \\
& +z_{j}^{3} g_{j, r} \sigma_{j}+z_{j}^{3} \hat{f}_{j, r}-\frac{3}{4} z_{j}^{4}+\frac{b_{K}}{4} z_{j+1}^{4}-\frac{b_{k}}{\ell_{j}} \tilde{\zeta}_{j} \dot{\hat{\zeta}}_{j}
\end{aligned}
$$


where $\hat{f}_{j, r}$ is defined as

$$
\begin{aligned}
\hat{f}_{j, r}= & f_{j, r}-L \sigma_{j-1}+\frac{3}{4} l_{j}^{-2} z_{j}\left\|\psi_{j, r}-\sum_{i=1}^{j-1} \frac{\partial \sigma_{j-1}}{\partial x_{i}} \psi_{i, r}\right\|^{4} \\
& +b_{K} z_{j}+\left(k_{j}+\frac{3}{4}\right) z_{j},
\end{aligned}
$$

with $k_{j}>0$ being a design parameter. Similarly, a $\operatorname{FLS} \Im_{j, r}^{T} \zeta_{j, r}\left(\bar{X}_{j}\right)$ is applied to approximating $\hat{f}_{j, r}$, where $\bar{X}_{j}=\left[\bar{x}_{j}^{T}, \hat{\zeta}_{j-1}, \bar{y}_{v}^{(j) T}\right]^{T} \in \Omega_{z_{j}} \subset R^{3 j}$ with $\overline{\hat{\zeta}}_{j-1}=\left[\hat{\zeta}_{1}, \hat{\zeta}_{2}, \ldots, \hat{\zeta}_{j-1}\right]^{T}$. From Lemma $4, \hat{f}_{j, r}$ satisfies

$$
\hat{f}_{j, r}=\Im_{j, r}^{T} \zeta_{j, r}\left(\bar{X}_{j}\right)+\xi_{j, r}\left(\bar{X}_{j}\right), \quad\left|\xi_{j, r}\left(\bar{X}_{j}\right)\right| \leq \epsilon_{j, r}
$$

where $\forall \epsilon_{j, r}>0$ is a constant. In addition, similar to (12), the following inequality can be got:

$$
z_{j}^{3} \hat{f}_{j, r} \leq \frac{b_{k}}{2 a_{j, r}^{2}} z_{j}^{6} s_{j} \zeta_{j, r}^{T} \zeta_{j, r}+\frac{1}{2} a_{j, r}^{2}+\frac{3}{4} z_{j}^{4}+\frac{1}{4} \epsilon_{j, r}^{4}
$$

where $\varsigma_{j}=\frac{\left\|\Im_{j}, r\right\|^{2}}{b_{k}}$ is an unknown constant and $a_{j, r}$ is a design parameter.

Then the virtual control signal and the adaptation law are constructed as

$$
\begin{aligned}
& \sigma_{j}=-k_{j} z_{j}-\frac{1}{2 a_{j, \min }^{2}} \hat{\zeta}_{j} z_{j}^{3} \zeta_{j, r}^{T} \zeta_{j, r}, \\
& \dot{\hat{\zeta}}_{j}=\frac{\ell_{j}}{2 a_{j, r}^{2}} z_{j}^{6} \zeta_{j, r}^{T} \zeta_{j, r}-\gamma_{j} \hat{\zeta}_{j}, \quad \hat{\zeta}_{j}(0) \geq 0,
\end{aligned}
$$

where $\gamma_{j}>0$ is a design constant. Similar to (15) and (17), it follows that

$$
\begin{gathered}
z_{j}^{3} h_{j, r} \sigma_{j} \leq-k_{j} b_{k} z_{j}^{4}-\frac{b_{k}}{2 a_{j, r}^{2}} z_{j}^{6} \hat{\zeta}_{j} \zeta_{j, r}^{T} \zeta_{j, r}, \\
\frac{b_{k} \gamma_{j}}{\ell_{j}} \tilde{\zeta}_{j} \hat{\zeta}_{j} \leq-\frac{b_{k} \gamma_{j}}{2 \ell_{j}} \tilde{\zeta}_{j}^{2}+\frac{b_{k} \gamma_{j}}{2 \ell_{j}} \varsigma_{j}^{2} .
\end{gathered}
$$

We substitute (33), (34), (35), (36), (37) into (32), and we have

$$
L V_{j} \leq-\sum_{i=1}^{j}\left(c_{i} z_{i}^{4}+\frac{b_{k} \gamma_{i}}{2 \ell_{i}} \tilde{\varsigma}_{i}^{2}\right)+\sum_{i=1}^{j} \varrho_{i}+\frac{b_{K}}{4} z_{j+1}^{4},
$$

where $c_{i}=k_{i}\left(1+b_{k}\right)$ and $\varrho_{i}=\frac{3}{4} l_{i}^{2}+\frac{1}{2} a_{i, r}^{2}+\frac{1}{4} \epsilon_{i, r}^{4}+\frac{b_{k} \gamma_{i}}{2 \ell_{i}} \varsigma_{i}^{2}, i=1,2, \ldots, j$.

Step $n$. By (5) and Itô's formula, it is deduced that

$$
\begin{aligned}
d z_{n}= & \left(h_{n, r} u_{\tau(t)}+f_{n, r}\left(\bar{x}_{n}\right)-L \sigma_{n-1}\right) d t \\
& +\left(\psi_{n, r}-\sum_{i=1}^{n-1} \frac{\partial \sigma_{n-1}}{\partial x_{i}} \psi_{i, r}\right)^{T} d \omega,
\end{aligned}
$$


where

$$
\begin{aligned}
L \sigma_{n-1}= & \sum_{i=1}^{n-1} \frac{\partial \sigma_{n-1}}{\partial x_{i}}\left[h_{i, r}\left(x_{i+1}\right)+f_{i, r}\left(\bar{x}_{i}\right)\right] \\
& +\sum_{i=1}^{n-1} \frac{\partial \sigma_{n-1}}{\partial \hat{\zeta}_{i}} \dot{\hat{\zeta}}_{i}+\sum_{i=0}^{n-1} \frac{\partial \sigma_{n-1}}{\partial y_{v}^{(i)}} y_{v}^{(i+1)} \\
& +\frac{1}{2} \sum_{p, q=1}^{n-1} \frac{\partial^{2} \sigma_{n-1}}{\partial x_{p} \partial x_{q}} \psi_{p, r}^{T} \psi_{q, r} .
\end{aligned}
$$

Consider the stochastic Lyapunov function of the form

$$
V_{n}=V_{n-1}+\frac{1}{4} z_{n}^{4}+\frac{b_{k}}{2 \ell_{n}} \tilde{\varsigma}_{n}^{2},
$$

where $\ell_{n}>0$ is a design constant.

Repeating a similar procedure to Step 1 , one has

$$
\begin{aligned}
L V_{n}= & L V_{n-1}+z_{n}^{3}\left[h_{n, r} u_{r}+f_{n, r}\left(\bar{x}_{n}\right)-L \sigma_{n-1}\right] \\
& +\frac{3}{2} z_{n}^{2}\left(\psi_{n, r}-\sum_{i=1}^{n-1} \frac{\partial \sigma_{n-1}}{\partial x_{i}} \psi_{i, r}\right)^{T}\left(\psi_{n, r}\right. \\
& \left.-\sum_{i=1}^{n-1} \frac{\partial \sigma_{n-1}}{\partial x_{i}} \psi_{i, r}\right)-\frac{b_{k}}{\ell_{n}} \tilde{\zeta}_{n} \dot{\hat{\zeta}}_{n} .
\end{aligned}
$$

Similar to (30), one can obtain

$$
\begin{aligned}
\frac{3}{2} z_{n}^{2}\left\|\psi_{n, r}-\sum_{i=1}^{n-1} \frac{\partial \sigma_{n-1}}{\partial x_{i}} \psi_{i, r}\right\|^{2} \leq & \frac{3}{4} l_{n}^{2} \\
& +\frac{3}{4} l_{n}^{-2} z_{n}^{4}\left\|\psi_{n, r}-\sum_{i=1}^{n-1} \frac{\partial \sigma_{n-1}}{\partial x_{i}} \psi_{i, r}\right\|^{4},
\end{aligned}
$$

where $l_{n}$ is a design constant. Substituting (38), (39), (40), (41), (43) into (42) yields

$$
\begin{aligned}
L V_{n} \leq & -\sum_{i=1}^{n-1}\left(c_{i} z_{i}^{4}+\frac{b_{k} \gamma_{i}}{2 \ell_{1}} \tilde{\zeta}_{i}^{2}\right)+\sum_{i=1}^{n-1} \varrho_{i}+\frac{3}{4} l_{n}^{2}-k_{n} z_{n}^{4} \\
& +z_{n}^{3} g_{n, r} u_{r}+z_{n}^{3} \hat{f}_{n, r}-\frac{3}{4} z_{n}^{4}-\frac{b_{k}}{\ell_{n}} \tilde{\zeta}_{n} \dot{\hat{\zeta}}_{n},
\end{aligned}
$$

where $\hat{f}_{n, r}$ is defined as

$$
\begin{aligned}
\hat{f}_{n, r}= & f_{n, r}+\frac{3}{4} l_{n}^{-2} z_{n}\left\|\psi_{n, r}-\sum_{i=1}^{n-1} \frac{\partial \sigma_{n-1}}{\partial x_{i}} \psi_{i, r}\right\|^{4} \\
& +\frac{1}{4} b_{K} z_{n}^{4}+\left(k_{n}+\frac{3}{4}\right) z_{n}^{4}-L \sigma_{n-1}
\end{aligned}
$$


with $k_{n}>0$ being a design parameter. Similarly, for $\forall \epsilon_{n, r}>0$, the unknown function $\hat{f}_{n, r}$ can be approximated by the FLS $\Im_{n, r}^{T} \zeta_{h, r}\left(\bar{X}_{n}\right)$. From Lemma 3, one has

$$
z_{n}^{3} \hat{f}_{n, r} \leq \frac{b_{k}}{2 a_{n, r}^{2}} z_{n}^{6} \varsigma_{n} \zeta_{n, r}^{T} \zeta_{n, r}+\frac{1}{2} a_{n, r}^{2}+\frac{3}{4} z_{n}^{4}+\frac{1}{4} \epsilon_{n, r}^{4}
$$

where $\varsigma_{j}=\frac{\left\|\widetilde{s}_{j},\right\|^{2}}{b_{k}}$ is an unknown constant and $a_{n, r}$ is a design parameter.

Then the control law and the adaptation law are designed as

$$
\begin{aligned}
& u_{r}=-k_{n} z_{n}-\frac{1}{2 a_{n, \min }^{2}} \hat{\zeta}_{n} z_{n}^{3} \zeta_{n, r}^{T} \zeta_{n, r}, \\
& \dot{\hat{\zeta}}_{n}=\frac{\ell_{n}}{2 a_{n, r}^{2}} z_{n}^{6} \zeta_{n, r}^{T} \zeta_{n, r}-\gamma_{n} \hat{\zeta}_{n}, \quad \hat{\zeta}_{n}(0) \geq 0,
\end{aligned}
$$

where $\gamma_{n}>0$ is a design constant.

According to (45), (46), (47) and Assumption 2, (44) can be written in the following form:

$$
L V_{n} \leq-\sum_{i=1}^{n}\left(c_{i} z_{i}^{4}+\frac{b_{k} \gamma_{i}}{2 \ell_{i}} \tilde{\zeta}_{i}^{2}\right)+\sum_{i=1}^{n} \varrho_{i},
$$

where $c_{i}=k_{i}\left(1+b_{k}\right)$ and $\varrho_{i}=\frac{3}{4} l_{i}^{2}+\frac{1}{2} a_{i, r}^{2}+\frac{1}{4} \epsilon_{i, r}^{4}+\frac{b_{k} \gamma_{i}}{2 \ell_{i}} \varsigma_{i}^{2}, i=1,2, \ldots, n$.

Defining $c=\min \left\{4 c_{i}, \gamma_{i}, i=1,2, \ldots, n\right\}$ and $w=\sum_{i=1}^{n} \varrho_{i}$, (47) becomes

$$
L V_{n} \leq-c V_{n}+w, \quad t \geq 0 .
$$

According to the definition of $V_{n}$ and Lemma $2, z_{i}$ and $\tilde{\zeta}_{i}$ are bounded in probability.

In addition, from [50], the following inequality is obtained:

$$
\frac{d E\left(V_{n}(t)\right)}{d t} \leq-c E\left(V_{n}(t)\right)+w, \quad t \geq 0,
$$

where $E(\cdot)$ indicates an expectation operator. Then it satisfies

$$
0 \leq E\left[V_{n}(t)\right] \leq\left(V_{n}(0)-\frac{w}{c}\right) e^{-c t}+\frac{w}{c}
$$

which means that

$$
E\left[V_{n}(t)\right] \leq \frac{w}{c}, \quad t \rightarrow \infty .
$$

From (51) and (52), we have

$$
E\left(\sum_{i=1}^{n} z_{i}^{4}\right) \leq 4 E\left[V_{n}(t) \leq \frac{4 w}{c}, \quad t \rightarrow \infty .\right.
$$

Therefore, $z_{i}$ eventually is convergent to the compact set $\Omega_{z}$ which is defined as

$$
\Omega_{z}=\left\{z_{i} \mid \sum_{i=1}^{n}\left[\left|z_{i}\right|^{4}\right] \leq \frac{4 w}{c}\right\} .
$$


Thus far, the design of adaptive fuzzy control based on backstepping technology has been completed. The main result will be presented by Theorem 1 .

Theorem 1 Consider a class switched stochastic nonlinear system (3), under Assumptions 1 and 2, for bounded initial conditions, parameter adaptive laws (35), the control law (46), and the intermediate control signals (47), guarantee that all the signals in the closed-loop system are SUUB and the tracking error is convergent to a neighborhood of the origin.

Remark 6 In [43], the adaptive tracking problem for a class of switched nonlinear systems was investigated. By combining the backstepping technique with the approximation scheme of FLS, a design approach with multiple adaptive laws was developed. In this paper, Theorem 1 generalizes the result of Theorem 1 in [43]. Considering the stochastic disturbances, the systems in this paper are more common.

\subsection{Adaptive control design under one adaptive law}

In this subsection, we will propose a controller design method with one adaptive law. For a stochastic pure-feedback system (3), according to

$$
z_{j}=x_{j}-\sigma_{j-1}
$$

the error dynamic is

$$
\begin{aligned}
d z_{j}= & {\left[h_{j, r}\left(x_{(j+1)}\right)+f_{j, r}\left(\bar{x}_{j-1}\right)-L \sigma_{1}\right] d t } \\
& +\left(\psi_{j, r}-\sum_{j=1}^{j-1} \frac{\partial \sigma_{j-1}}{\partial x_{i}} \psi_{i, r}\right)^{T} d \omega,
\end{aligned}
$$

where

$$
\begin{aligned}
L \sigma_{j-1}= & \sum_{i=1}^{j-1} \frac{\partial \sigma_{j-1}}{\partial x_{i}}\left[h_{i, r}\left(x_{i+1}\right)+f_{i, r}\left(\bar{x}_{i}\right)\right] \\
& +\sum_{i=1}^{j-1} \frac{\partial \sigma_{j-1}}{\partial \hat{\zeta}} \dot{\hat{\zeta}}+\sum_{i=0}^{j-1} \frac{\partial \sigma_{j-1}}{\partial y_{v}^{(i)}} y_{v}^{(i+1)} \\
& +\frac{1}{2} \sum_{p, q=1}^{j-1} \frac{\partial^{2} \sigma_{j-1}}{\partial x_{p} \partial x_{q}} \psi_{p, r}^{T} \psi_{q, r} .
\end{aligned}
$$

A stochastic Lyapunov function is taken as

$$
V=\sum_{i=1}^{n} \frac{1}{4} z_{i}^{4}+\frac{b_{k}}{2 \ell} \tilde{\zeta}^{2}
$$

where $\ell>0$ is a design constant, and $\tilde{\zeta}=\varsigma-\hat{\zeta}$ is a parameter error, define a constant $\varsigma=\frac{\left\|\widetilde{s}_{n, r}\right\|^{2}}{b_{k}}$.

Applying (2), (55) and (56), one has

$$
L V=\sum_{j=1}^{n-1}\left[z_{j}^{3}\left(h_{j, r}\left(z_{j+1}+\sigma_{j}\right)+f_{j, r}\left(\bar{x}_{j}\right)-L \sigma_{j-1}\right)\right.
$$




$$
\begin{aligned}
& +\frac{3}{2} z_{j}^{2}\left(\psi_{j}-\sum_{i=1}^{j-1} \frac{\partial \sigma_{j-1}}{\partial x_{i}} \psi_{i}\right)^{T}\left(\psi_{j}-\sum_{i=1}^{j-1} \frac{\partial \sigma_{j-1}}{\partial x_{i}} \psi_{i}\right) \\
& \left.-\frac{b_{m}}{\ell} \widetilde{\varsigma} \widehat{\varsigma}\right]+z_{n}^{3}\left(g_{n, r} u_{r}+f_{n, r}\left(\bar{x}_{n}\right)-L \sigma_{n-1}\right) \\
& +\frac{3}{2} z_{n}^{2}\left(\psi_{n}-\sum_{i=1}^{n-1} \frac{\partial \sigma_{n-1}}{\partial x_{i}} \psi_{i}\right)^{T}\left(\psi_{n}-\sum_{i=1}^{n-1} \frac{\partial \sigma_{n-1}}{\partial x_{i}} \psi_{i}\right) \\
& -\frac{b_{m}}{\ell} \widetilde{\varsigma} \widehat{\varsigma} .
\end{aligned}
$$

Based on Lemma 3 and Assumption 2, the following inequalities are obtained:

$$
\begin{aligned}
\frac{3}{2} z_{j}^{2}\left\|\psi_{j, r}-\sum_{i=1}^{j-1} \frac{\partial \sigma_{j-1}}{\partial x_{i}} \psi_{i, r}\right\| \leq & \frac{3}{4} l_{j}^{2} \\
& +\frac{3}{4} l_{j}^{-2} z_{j}^{4}\left\|\psi_{j, r}-\frac{\partial \sigma_{j-1}}{\partial x_{i}} \psi_{i, r}\right\|^{4} \\
h_{j, r} z_{j}^{3} z_{j+1} \leq & \frac{3}{4} b_{K} z_{j}^{4}+\frac{b_{K}}{4} z_{j+1}^{4},
\end{aligned}
$$

where $l_{j}$ is a design constant.

Design the control laws as

$$
u_{r}=-k_{n} z_{n}-\frac{1}{2 a_{n, \min }^{2}} \hat{\varsigma} z_{n}^{3} \zeta_{n, r}^{T} \zeta_{n, r}
$$

where $a_{n, \min }^{2}=\min \left\{a_{n, r}: r \in M\right\}$ and $a_{n, r}>0$ is design parameter, $k_{n}>0$ is a design constant, $\hat{\zeta}$ is the estimation of $\varsigma=\sum_{j=1}^{n} \frac{\left\|\Im_{j, r}\right\|^{2}}{b_{k}}, \Im_{j, \max }=\max \left\{\Im_{j, r}: r \in M\right\}$ and the unknown function $\hat{f}_{j, r}(\bar{X})$ can be approximated by $\mathfrak{\Im}_{n, r}$ in FLS $\Im_{n, r}^{T} \zeta_{n, r}(\bar{X})$.

The adaptive law is defined as the solution of differential equation as follows:

$$
\dot{\hat{\zeta}}=\frac{\ell}{2 a_{i, r}^{2}} z_{i}^{6} \zeta_{i, r}^{T} \zeta_{i, r}-\gamma \hat{\zeta},
$$

where $\ell, a_{i, r}>0$ and $\gamma_{i}>0$ are design parameters, $a_{i, \min }^{2}=\min \left\{a_{n, r}: r \in M\right\}$ and the selection of $\hat{\zeta}$ is needed to satisfy $\hat{\zeta}(0) \geq 0$ such that $\hat{\zeta} \geq 0$.

Define the functions as

$$
\begin{aligned}
& \hat{f}_{j, r}=f_{j, r}-L \sigma_{j-1}+\frac{3}{4} l_{j}^{-2} z_{n}\left\|\psi_{j}-\sum_{i=1}^{j-1} \frac{\partial \sigma_{j-1}}{\partial x_{i}} \psi_{i}\right\|^{4} \\
& \hat{f}_{n, r}=f_{n, r}-L \sigma_{n-1}+\frac{3}{4} l_{n}^{-2} z_{n}\left\|\psi_{n}-\sum_{i=1}^{n-1} \frac{\partial \sigma_{n-1}}{\partial x_{i}} \psi_{i}\right\|^{4} \\
& \quad+\frac{3}{4} b_{k} z_{n}^{4} .
\end{aligned}
$$


Substituting (58), (59), (60), (61), (62), (63) into (57) gives

$$
L V_{n} \leq \sum_{i=1}^{n}\left(c_{i} z_{i}^{4}+\frac{b_{k} \gamma}{2 \ell} \tilde{\varsigma}^{2}\right)+\sum_{i=1}^{n} \varrho_{i}
$$

where $c_{i}=\left(-k_{i} b_{k}+b_{K}+\frac{3}{4}\right) z_{n}^{4}$ and $\varrho_{i}=\frac{3}{4} l_{i}^{2}+\frac{1}{2} a_{i, r}^{2}+\frac{1}{4} \epsilon_{i, r}^{2}+\frac{b_{k} \gamma_{i}}{2 \ell_{i}} \varsigma^{2}, i=1,2, \ldots, n$. The remaining part of the proof is similar to (48), (49), (50), (51), (52), (53), (54), which is omitted here.

Theorem 2 Consider a class switched stochastic nonlinear system (3), under Assumptions 1 and 2, for bounded initial conditions, the control law (60), and the intermediate control signals (61), guarantee that all the signals in the closed-loop system are SUUB and the tracking error converging to a neighborhood of the origin.

Remark 7 In [43], the adaptive tracking problem for a class of switched nonlinear systems was investigated. By combining the backstepping technique with the approximation approach of FLS, a design scheme with only one adaptive laws was developed. In this paper, it is noted that Theorem 2 generalizes the result of Theorem 2 in [43].

\section{Simulation example}

In this section, a simulation example is proposed in order to certify the control performance and the feasibility of the presented method in the previous sections.

Example 1 Given the following second-order stochastic nonlinear switched systems:

$$
\begin{aligned}
& d x_{1}=\left[h_{1, \tau(t)} x_{2}+f_{1, \tau(t)}\left(\bar{x}_{1}\right)\right] d t+\psi_{1, \tau(t)}\left(\bar{x}_{1}\right) d \omega, \\
& d x_{2}=\left[h_{2, \tau(t)} u_{\tau(t)}+f_{2, \tau(t)}\left(\bar{x}_{2}\right)\right] d t+\psi_{2, \tau}\left(\bar{x}_{2}\right) d \omega, \\
& y=x_{1},
\end{aligned}
$$

where $\tau(t):[0, \infty] \rightarrow\{1,2\}, h_{11}=2, h_{12}=1, h_{21}=2, h_{22}=1, f_{12}=2 x_{1} \cos \left(x_{1}\right), f_{21}=$ $\left(x_{1}\right)^{2} \cos ^{2}\left(x_{2}\right), f_{11}=x_{1}, f_{22}=2 \sin ^{2}\left(x_{1}\right) x_{2}^{2}, \psi_{11}=\frac{x_{1}^{2}}{1+x_{1}^{2}}, \psi_{12}=\frac{0.03 x_{1}^{2}}{1+x_{1}^{2}}, \psi_{21}=0.6 \sin \left(2 x_{1} x_{2}\right), \psi_{22}=$ $\frac{0.05 x_{2}^{2}}{1+x_{1}^{2}}$. The purpose of control is to design a common adaptive fuzzy controller $u_{k}$ such that all signals in the closed-loop are bounded in probability and $y$ follows a desired reference signal $y_{v}$ under arbitrary switchings, where $y_{v}=\sin t$. In the simulation, first, we design the controllers under multiple adaptive laws by Theorem 1 . The initial conditions are $x_{1}(0)=0.05, x_{2}(0)=0.05, x_{3}=0$, and $\hat{\zeta}_{1}(0)=\hat{\zeta}_{2}(0)=0$. We choose $k_{1}=2, k_{2}=1, a_{1,1}=0.25$, $a_{1,2}=3, a_{2,1}=0.3, a_{2,2}=2.5, \ell_{1}=10, \ell_{2}=3, \gamma_{1}=\gamma_{2}=0.02$. Second, we design the controller under one adaptive law by Theorem 2 . The initial conditions are taken as $x_{1}(0)=0.1$, $x_{2}(0)=0.05, x_{3}=0.5, \hat{\zeta}(0)=0.5$. We choose $k_{1}=2, k_{2}=1, a_{1,1}=0.25, a_{1,2}=3, a_{2,1}=1.5$, $a_{2,2}=2, \ell=2, \gamma=0.025$.

Choose the following membership functions which are used to construct the fuzzy controller:

$$
\begin{aligned}
& \mu_{F_{j}^{1}}\left(x_{j}\right)=\exp \left(-0.5(x+3)^{2}\right), \\
& \mu_{F_{j}^{1}}\left(x_{j}\right)=\exp \left(-0.5(x+2)^{2}\right),
\end{aligned}
$$




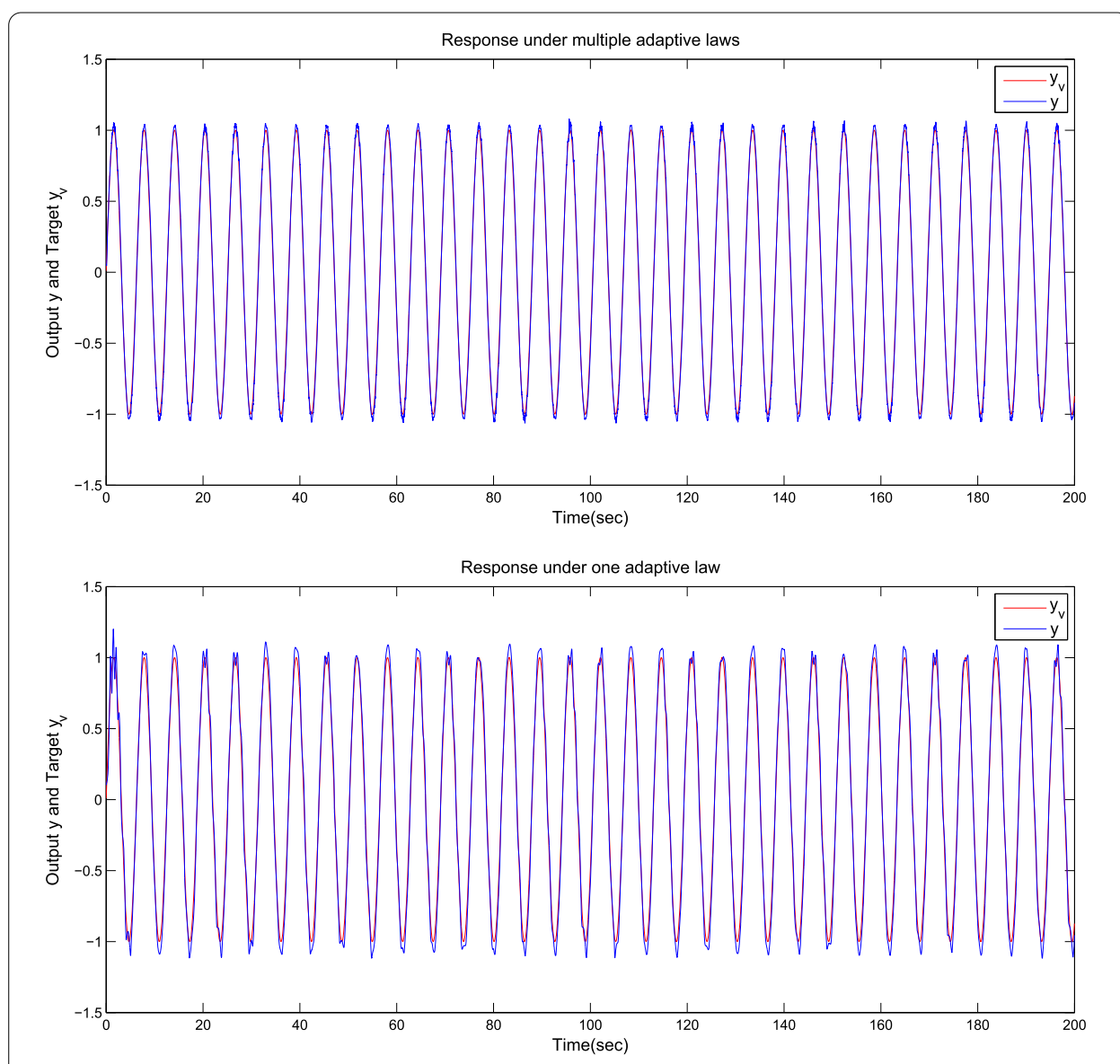

Figure 1 Tracking performances of Example 1

$$
\begin{aligned}
& \mu_{F_{j}^{1}}\left(x_{j}\right)=\exp \left(-0.5(x+1)^{2}\right), \\
& \mu_{F_{j}^{1}}\left(x_{j}\right)=\exp \left(-0.5 x^{2}\right), \\
& \mu_{F_{j}^{1}}\left(x_{j}\right)=\exp \left(-0.5(x-1)^{2}\right), \\
& \mu_{F_{j}^{1}}\left(x_{j}\right)=\exp \left(-0.5(x-2)^{2}\right), \\
& \mu_{F_{j}^{1}}\left(x_{j}\right)=\exp \left(-0.5(x-3)^{2}\right) .
\end{aligned}
$$

According to Theorem 1, $\hat{\zeta}_{1}, \hat{\zeta}_{2}$ and $u_{r}$ are selected, respectively, thus:

$$
\begin{aligned}
& \dot{\hat{\zeta}}_{1}=\frac{\ell_{1}}{2 a_{1,1}^{2}} z_{1}^{6} \zeta_{1,1}^{T} \zeta_{1,1}-\gamma_{1} \hat{\zeta}_{1}, \\
& \dot{\hat{\zeta}}_{2}=\frac{\ell_{2}}{2 a_{2,1}^{2}} z_{2}^{6} \zeta_{2,1}^{T} \zeta_{2,1}-\gamma_{2} \hat{\zeta}_{2}, \\
& u_{1}=-k_{2} z_{2}-\frac{1}{2 a_{2,1}^{2}} \hat{\zeta}_{2} z_{2}^{3} \zeta_{2,1}^{T} \zeta_{2,1}, \\
& u_{2}=-k_{2} z_{2}-\frac{1}{2 a_{2,2}^{2}} \hat{\zeta}_{2} z_{2}^{3} \zeta_{2,2}^{T} \zeta_{2,2},
\end{aligned}
$$

where $z_{1}=x_{1}-y_{v}, z_{2}=x_{2}-\sigma_{1}$. 


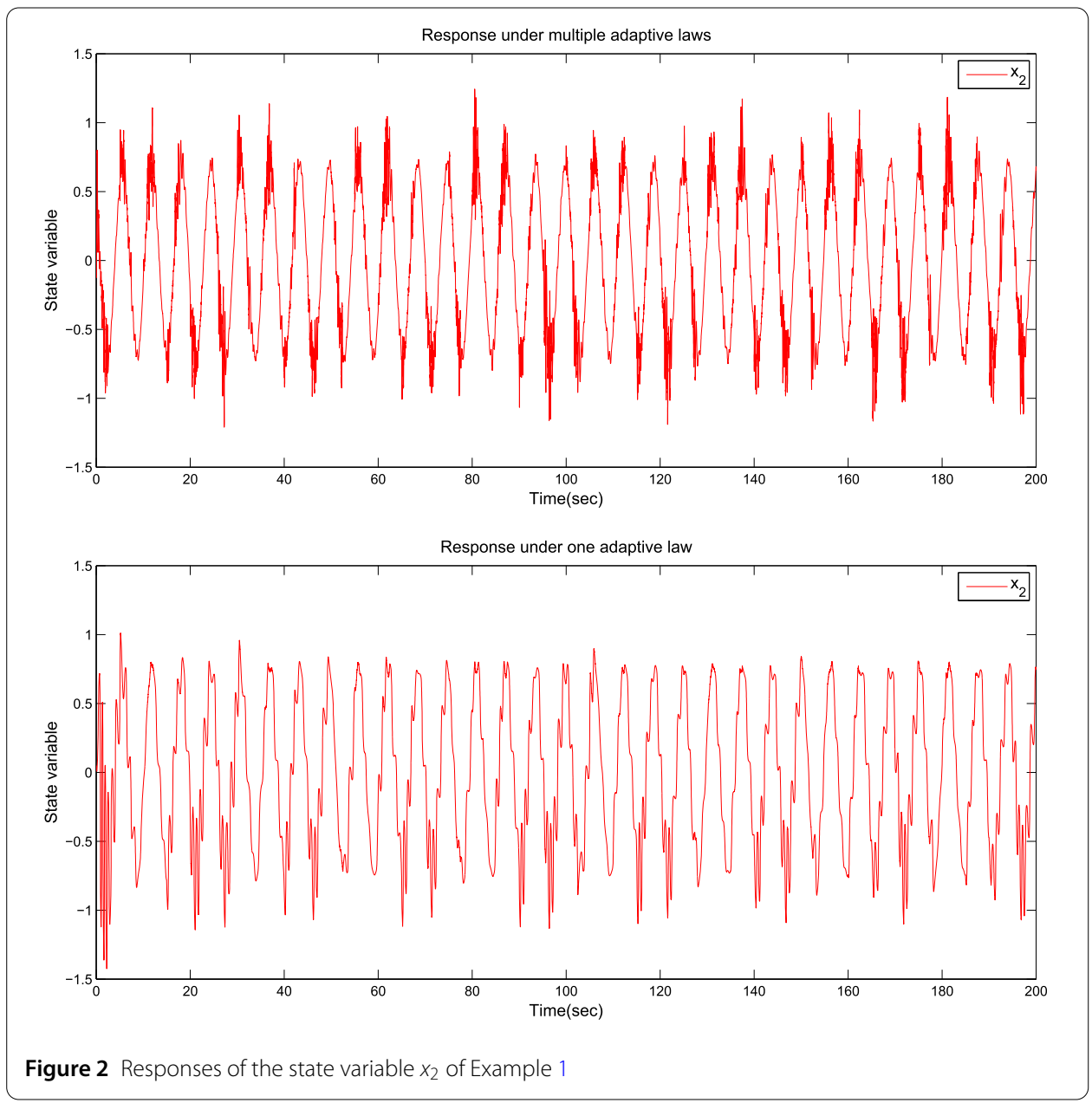

The virtual control function $\sigma_{1}$ is defined by

$$
\sigma_{1}=-k_{1} z_{1}-\frac{1}{2 a_{1,1}^{2}} \hat{\zeta}_{1} z_{1}^{3} \zeta_{1,1}^{T} \zeta_{1,1}
$$

The above is the controller design based on Theorem 1. Next, another design based on Theorem 2 is proposed. According to Theorem 2, $\hat{\zeta}$ and $u_{r}$ are selected, respectively, as

$$
\begin{aligned}
& \dot{\hat{\zeta}}=\frac{\ell}{2 a_{1,1}^{2}} z_{1}^{6} \zeta_{1,1}^{T} \zeta_{1,1}+\frac{\ell}{2 a_{2,1}^{2}} z_{2}^{6} \zeta_{2,1}^{T} \zeta_{2,1}-\gamma \hat{\zeta}, \\
& u_{1}=-k_{2} z_{2}-\frac{1}{2 a_{2,1}^{2}} \hat{\zeta}_{2} z_{2}^{3} \zeta_{2,1}^{T} \zeta_{2,1}, \\
& u_{2}=-k_{2} z_{2}-\frac{1}{2 a_{2,2}^{2}} \hat{\zeta}_{2} z_{2}^{3} \zeta_{2,2}^{T} \zeta_{2,2},
\end{aligned}
$$

where $z_{1}=x_{1}-y_{v}, z_{2}=x_{2}-\sigma_{1}$.

The virtual control function $\sigma_{1}$ is defined by

$$
\sigma_{1}=-k_{1} z_{1}-\frac{1}{2 a_{1,1}^{2}} \hat{\zeta} z_{1}^{3} \zeta_{1,1}^{T} \zeta_{1,1}
$$



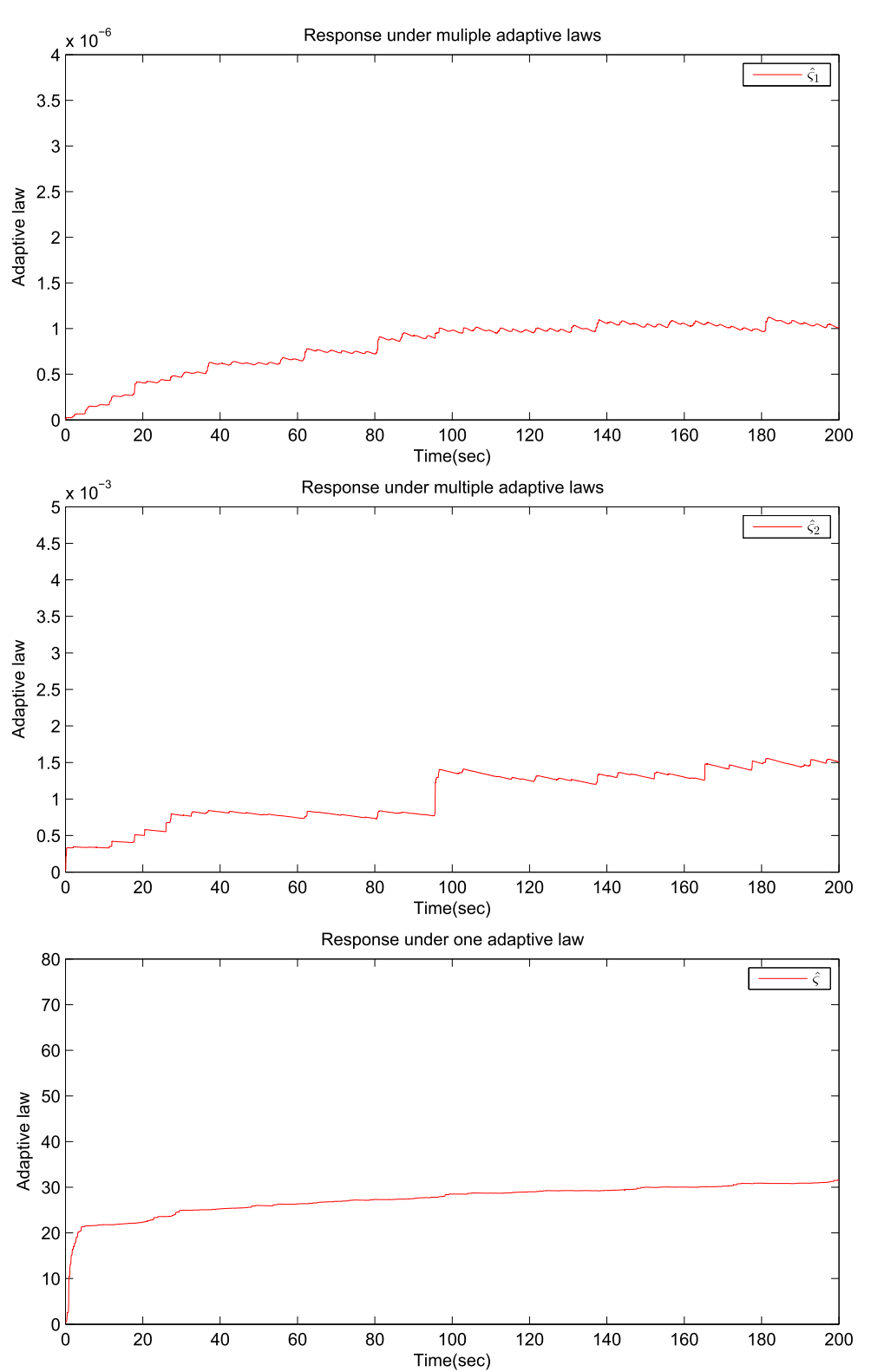

Figure 3 Responses of the adaptive laws of Example 1

The simulation results are shown in Figs. 1-4, respectively. Figure 1 demonstrates the system output $y$ and reference signal $y_{v}$. Figure 2 exhibits the trajectory of the state variable $x_{2}$. Figure 3 illustrates the trajectory of adaptive law. Figure 4 displays he trajectory of the control signal $u_{k}$. Figure 5 certifies the evolution of switching signal. From Fig. 1, Fig. 2, Fig. 3, it can be seen that the output $y$ of both controllers can track the target signal $y_{v}$ well, and all the closed-loop signals remain bounded.

Remark 8 For the same initial conditions, simulations were run by using the two controllers mentioned above. Figure 1 shows the tracking performances of the two adaptive controllers proposed in this paper, respectively. Figure 6 shows the tracking error under 

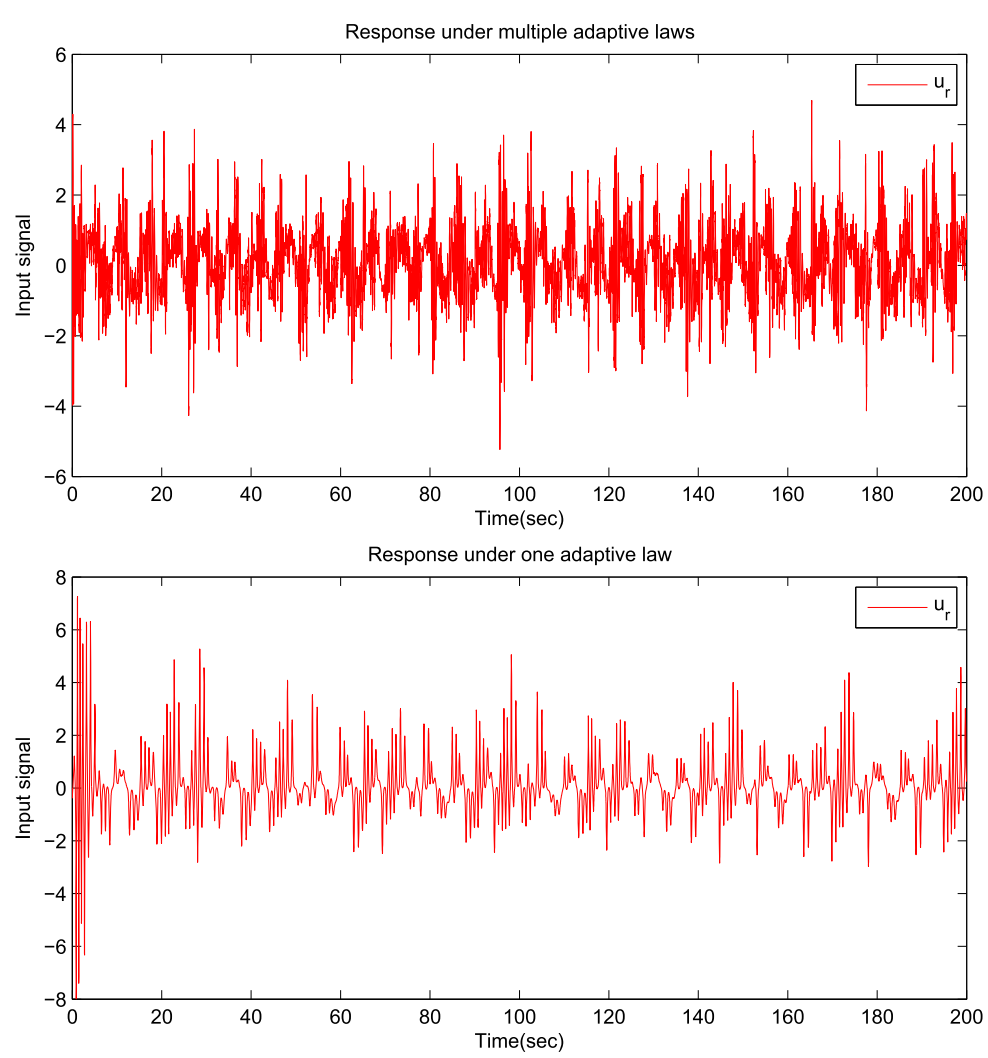

Figure 4 Responses of the control input signal of Example 1

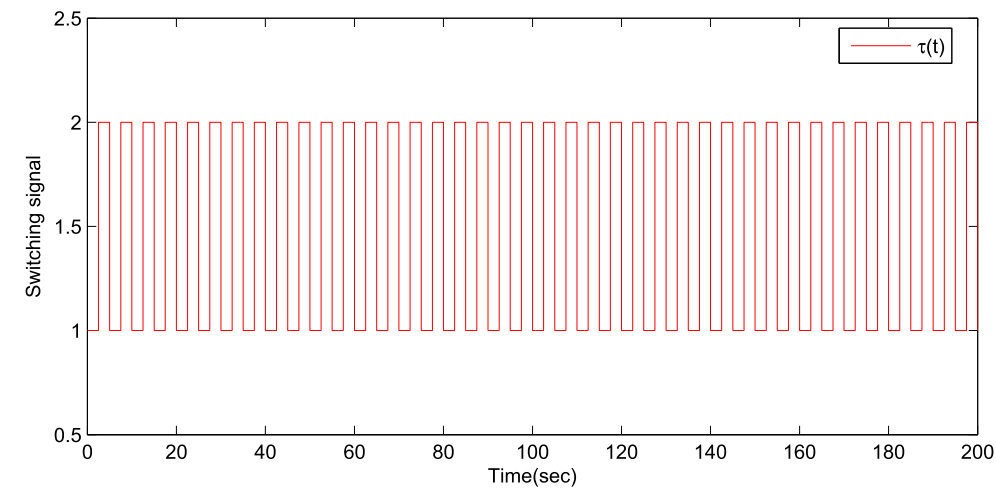

Figure 5 Responses of the control switching signal of Example 1

the action of two adaptive controllers. From Figs. 1 and 6, it can be seen that two controllers proposed in this paper can achieve the system stability, but the controllers in Theorem 1 work better than the one proposed in Theorem 2 . The main reason may be that the control design process in Theorem 2 cannot deal with the mismatching nonlinear term well. 

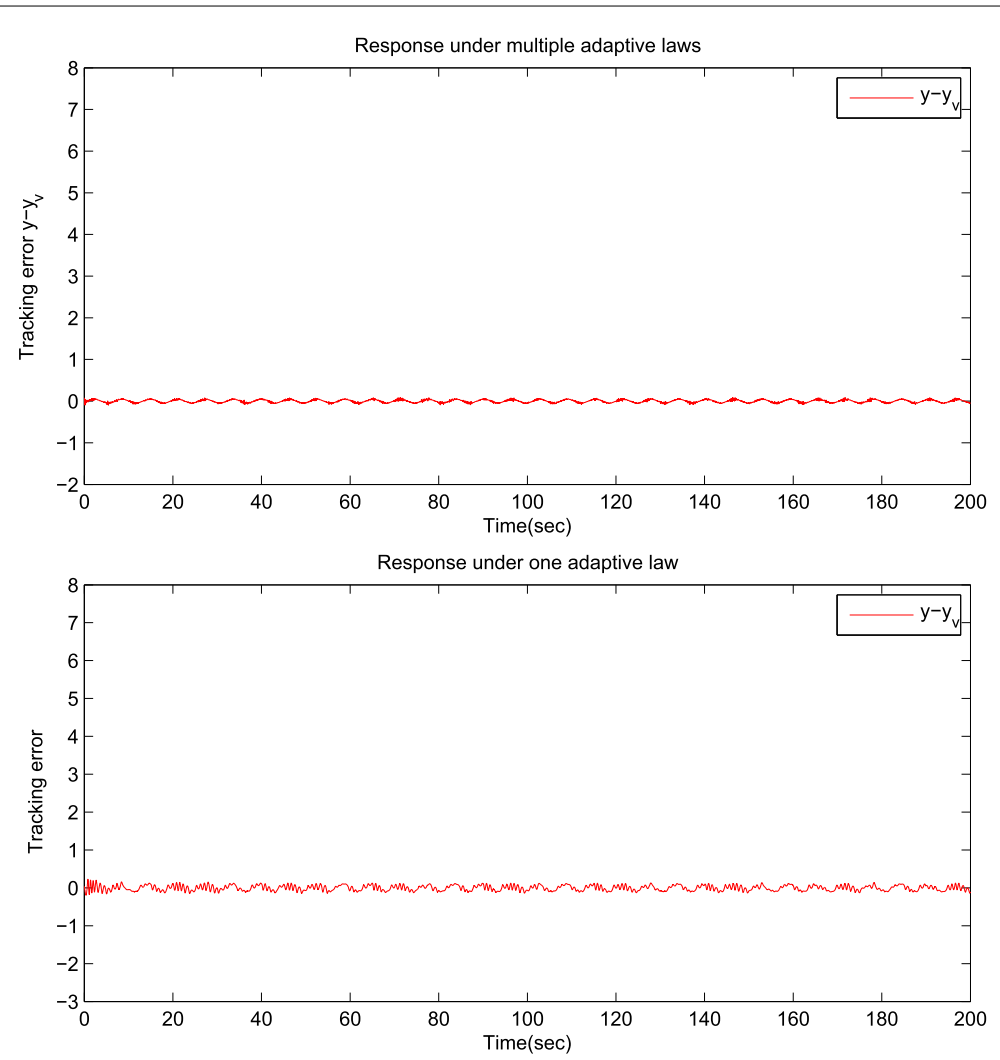

Figure 6 Responses of the tracking error of Example 1

Example 2 Some continuous stirred tank reactor with two modes feed stream can be modeled as the following switched system after some manipulations [51]:

$$
\left\{\begin{array}{l}
\dot{x}_{1}=x_{2}+f_{\sigma(t), 1}\left(x_{1}\right), \\
\dot{x}_{2}=u,
\end{array}\right.
$$

where $\sigma:[0,+\infty) \rightarrow 1,2, f_{1,1}\left(x_{1}\right)=0.5 x_{1}$ and $f_{1,2}\left(x_{1}\right)=2 x_{1}$. Further, it is supposed that there exists multiplicative white noise in the above system due to $f_{\sigma(t), 1}$, and as a result, we have the following stochastic nonlinear system:

$$
\begin{aligned}
& d x_{1}=\left[h_{1, \tau(t)} x_{2}+f_{1, \tau(t)}\left(\bar{x}_{1}\right)\right] d t+\psi_{1, \tau(t)}\left(\bar{x}_{1}\right) d \omega, \\
& d x_{2}=u_{\tau(t)} d t, \\
& y=x_{1},
\end{aligned}
$$

where $\tau(t):[0, \infty] \rightarrow\{1,2\}, h_{11}=2, h_{12}=1, f_{11}=x_{1}, f_{12}=2 x_{1} \cos \left(x_{1}\right), \psi_{11}=\frac{x_{1}^{2}}{1+x_{1}^{2}}, \psi_{12}=$ $\frac{0.03 x_{1}^{2}}{1+x_{1}^{2}}$. In the simulation, choose the fuzzy systems as those in Example 1 and the reference signal $y_{v}=\sin t$. The initial conditions are $x_{1}(0)=0.05, x_{2}(0)=0.05, x_{3}=0$, and $\hat{\zeta}_{1}(0)=$ $\hat{\zeta}_{2}(0)=0$. We choose $k_{1}=2, k_{2}=1, a_{1,1}=0.25, a_{1,2}=3, a_{2,1}=0.3, a_{2,2}=2.5, \ell_{1}=10, \ell_{2}=$ $3, \gamma_{1}=\gamma_{2}=0.02$. Figures $7-11$ show the simulation results. Figure 7 shows the tracking 


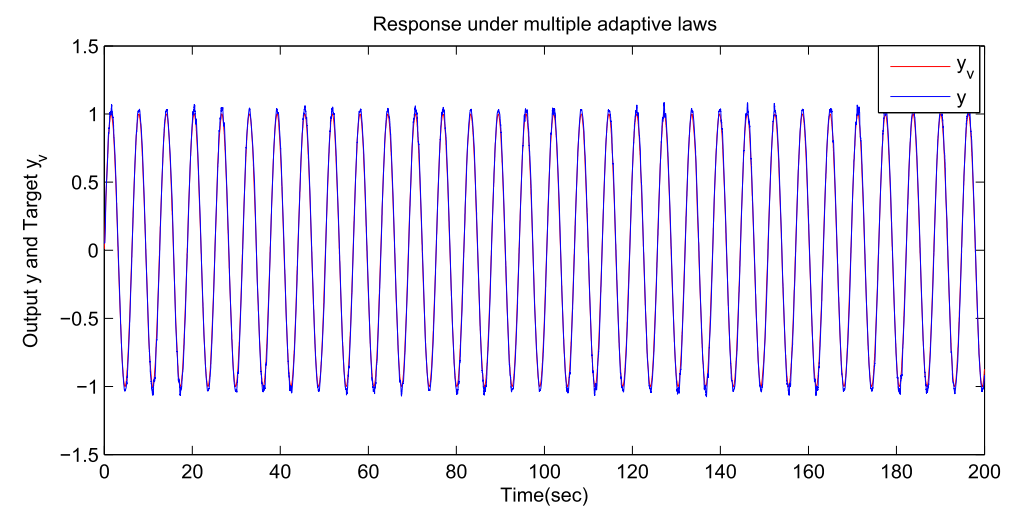

Figure 7 Tracking performances of Example 2

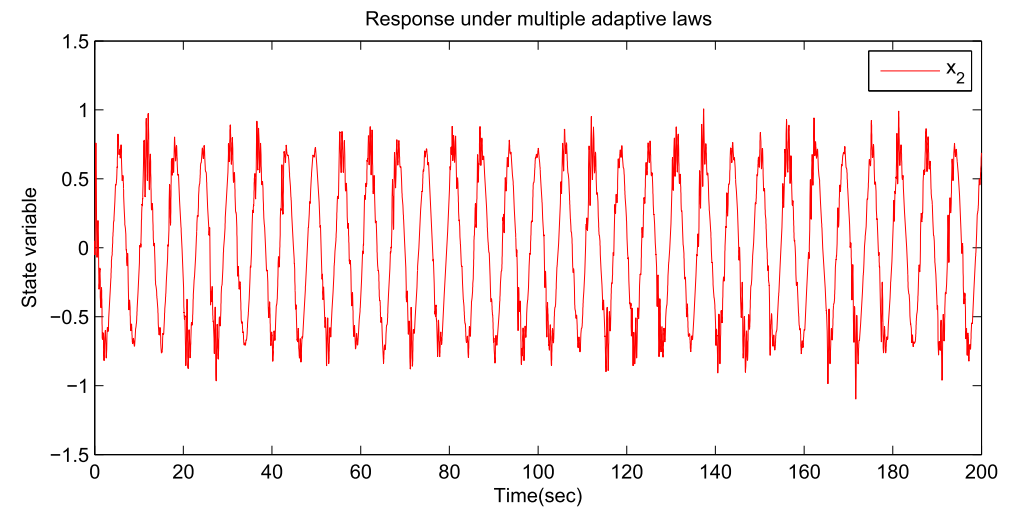

Figure 8 Responses of the state variable $x_{2}$ of Example 2

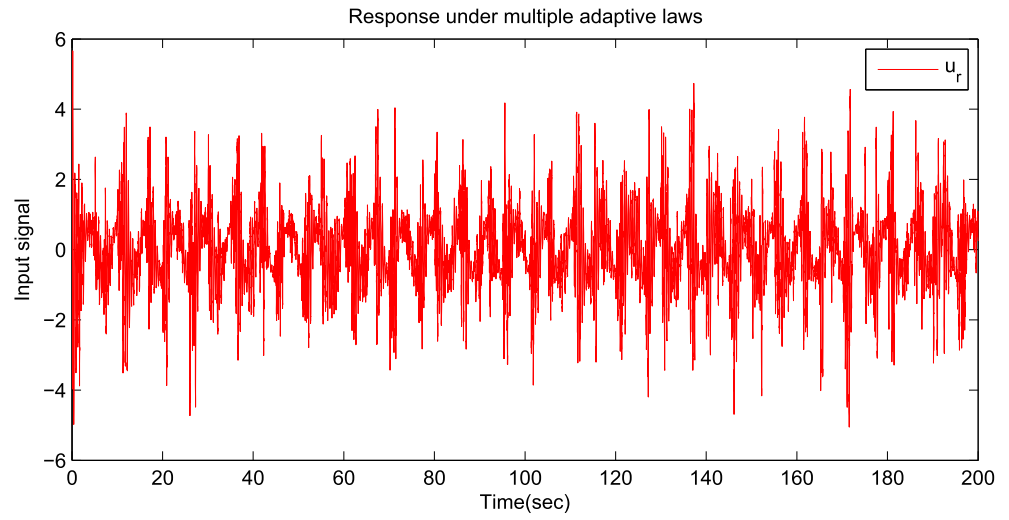

Figure 9 Responses of the control input signal of Example 2

results. Figures $8-10$ show that the variables $x_{2}, u_{r}$ and $\hat{\zeta}$ are bounded. Figure 11 shows the switching signal. 

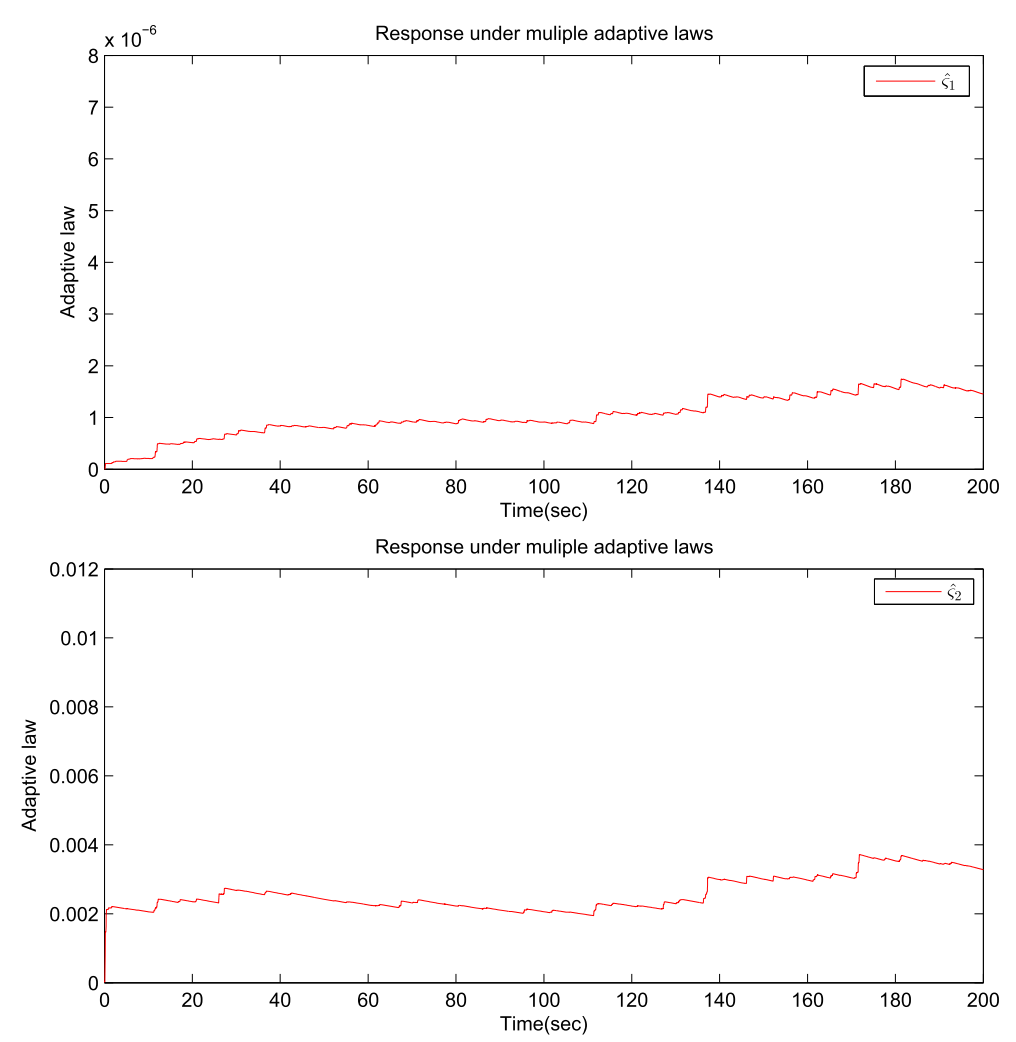

Figure 10 Responses of the adaptive laws of Example 2

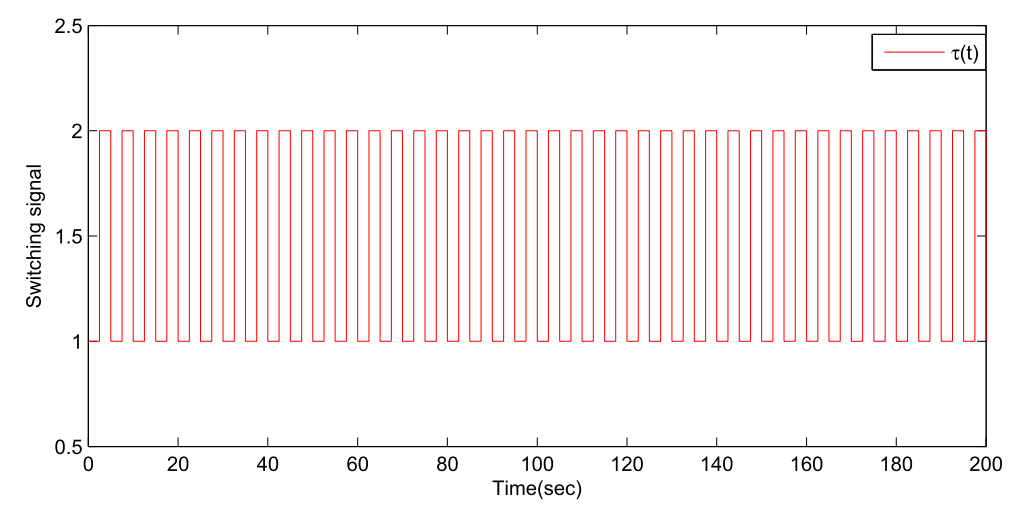

Figure 11 Responses of the control switching signal of Example 2

\section{Conclusion}

This paper studied the adaptive tracking control problem for a class of stochastic nonlinear systems under arbitrary switchings. It was noted that the nonlinear functions and stochastic disturbances of the system were completely unknown. For the sake of releasing the computational burden, the unknown nonlinear function of the system was estimated by employing the approximation property of FLS, then the adaptive backstepping technique was used to construct a class of adaptive fuzzy control. Under arbitrary switching conditions, the presented controller could ensure that all the signals in the closed-loop system 
remained bounded in probability and the system output converged to a small neighborhood of the reference signal. Finally, simulation results further showed the effectiveness of the proposed approaches.

\section{Acknowledgements}

We are thankful to the reviewers for their useful corrections and suggestions, which improved the quality of this paper.

\section{Funding}

This research work has been supported financially by the National Natural Science Foundation of China (Grant no. 61402265, 61573227), Shandong Provincial Natural Science Foundation (No. ZR2018MF013, ZR2016FM48), the Research Fund for the Taishan Scholar Project of Shandong Province of China, SDUST Research Fund (No. 2015TDJH105) and the Fund for Postdoctoral Application Research Project of Qingdao (01020120607).

\section{Competing interests}

The authors declare that they have no competing interests.

\section{Authors' contributions}

The main idea of this paper was proposed by the first and last authors, while the second and last authors reviewed and modified the paper. Furthermore, all authors read and approved the final manuscript.

\section{Author details}

${ }^{1}$ College of Mathematics and Systems Science, Shandong University of Science and Technology, Qingdao, China.

${ }^{2}$ College of Electrical Engineering and Automation, Shandong University of Science and Technology, Qingdao, China.

\section{Publisher's Note}

Springer Nature remains neutral with regard to jurisdictional claims in published maps and institutional affiliations.

Received: 4 July 2018 Accepted: 16 January 2019 Published online: 28 January 2019

\section{References}

1. Vu, L., Liberzon, D.: Common Lyapunov functions for families of commuting nonlinear systems. Syst. Control Lett. 54, 405-416 (2005)

2. Huang, L.T., Li, Y.M., Tong, S.C.: Fuzzy adaptive output feedback control for a class of switched non-triangular structure nonlinear systems with time-varying delays. Int. J. Syst. Sci. 49(1), 132-146 (2018)

3. Briat, C., Seuret, A.: Convex dwell-time characterizations for uncertain linear impulsive systems. IEEE Trans. Autom. Control 57(12), 3241-3246 (2012)

4. Briat, C., Seuret, A.: Affine characterizations of minimal and mode-dependent dwell-times for uncertain linear switched systems. IEEE Trans. Autom. Control 58(5), 1304-1310 (2013)

5. Xiang, W., Xiao, J.: Stabilization of switched continuous-time systems with all modes unstable via dwelltime switching. Automatica 50(2), 940-945 (2014)

6. Liu, Y.J., Lu, S., Tong, S., Chen, X.: Adaptive control-based Barrier Lyapunov functions for a class of stochastic nonlinear systems with full state constraints. Automatica 87, 83-93 (2018)

7. Pan, Z.G., Basar, T.: Adaptive controller design for tracking and disturbance attenuation in parametric strict-feedback nonlinear systems. IEEE Trans. Autom. Control 43(8), 1066-1083 (1998)

8. Ma, R.C., Zhao, J.: Backstepping design for global stabilization of switched nonlinear systems in lower triangular form under arbitrary switchings. Automatica 46(11), 1819-1823 (2010)

9. Niu, B., Zhao, J.: Tracking control for output-constrained nonlinear switched systems with a barrier Lyapunov function. Int. J. Syst. Sci. 44(5), 978-985 (2013)

10. Krstic, M., Kanellakopoulos, I., Kokotovic, P.V.: Nonlinear and Adaptive Control Design. Wiley, New York (1995)

11. Long, L.J., Zhao, J.: $H_{\infty}$ control of switched nonlinear systems in p-normal form using multiple Lyapunov functions. IEEE Trans. Autom. Control 57, 1285-1291 (2012)

12. Liberzon, D., Morse, A.S.: Basic problems in stability and design of switched systems. IEEE Control Syst. Mag. 19(5), 59-70 (1999)

13. Homaee, O., Zakariazadeh, A., Jadid, S.: Real-time voltage control algorithm with switched capacitors in smart distribution system in presence of renewable generations. Int. J. Electr. Power Energy Syst. 54(1), 187-197 (2014)

14. Yang, H., Jiang, B., Cocquempot, V.: A survey of results and perspectives on stabilization of switched nonlinear systems with unstable modes. Nonlinear Anal. Hybrid Syst. 13, 45-60 (2014)

15. Wang, M., Zhao, J.: Quadratic stabilization of a class of switched nonlinear systems via single Lyapunov function. Nonlinear Anal. Hybrid Syst. 4(1), 44-53 (2010)

16. Xi, C.J., Zhai, D., Dong, J.X., Zhang, Q.L.: Approximation-based adaptive fuzzy tracking control for a class of switched nonlinear pure-feedback systems. Int. J. Syst. Sci. 48(12), 2463-2472 (2017)

17. Si, W.J., Dong, X.D.: Adaptive neural DSC for stochastic nonlinear constrained systems under arbitrary switchings. Nonlinear Dyn. 12,1-14 (2017)

18. Zhao, X.D., Liu, X.W., Yin, S., Li, H.Y.: Improved results on stability of continuous-time switched positive linear systems. Automatica 50, 614-621 (2014)

19. Zhao, X.D., Yin, S., Li, H.Y., Niu, B.: Switching stabilization for a class of slowly switched systems. IEEE Trans. Autom. Control 60(1), 221-226 (2015)

20. Zhao, X.D., Zhang, L.X., Shi, P., Liu, M.: Stability and stabilization of switched linear systems with mode-dependent average dwell time. IEEE Trans. Autom. Control 57, 1809-1815 (2012) 
21. Liu, X.K., Li, Y., Zhang, W.H.: Stochastic linear quadratic optimal control with constraint for discrete-time systems. Appl. Math. Comput. 228, 264-270 (2004)

22. Li, Y., Liu, X.K.: H_ index for nonlinear stochastic systems with state and input dependent noises. Int. J. Fuzzy Syst. 20, 759-768 (2018)

23. Li, Y., Zhang, W.H., Liu, X.K.: H_ index for discrete-time stochastic systems with Markovian jump and multiplicative noise. Automatica 90, 268-293 (2018)

24. Liu, Y.L., Ma, H.J.: Adaptive fuzzy tracking control of nonlinear switched stochastic systems with prescribed performance and unknown control directions. IEEE Trans. Syst. Man Cybern. 99, 1-10 (2017)

25. Yin, Y., Shi, P., Liu, F.: Gain-scheduled robust fault detection on time-delay stochastic nonlinear systems. IEEE Trans. Ind. Electron. 58(10), 4908-4916 (2011)

26. Li, W., Jing, Y., Zhang, S.: Adaptive state-feedback stabilization for a large class of high-order stochastic nonlinear systems. Automatica 47(4), 819-828 (2011)

27. Zhou, Q., Shi, P.: A new approach to network-based $H_{\infty}$ control for stochastic systems. Int. J. Robust Nonlinear Control 22(9), 1036-1059 (2012)

28. Wang, H., Liu, P.X., Niu, B.: Robust fuzzy adaptive tracking control for nonaffine stochastic nonlinear switching systems. IEEE Trans. Cybern. 99, 1-10 (2018)

29. Liu, Z., Wang, F., Zhang, Y., Chen, X., Philip Chen, C.L.: Adaptive tracking control for a class of nonlinear systems with fuzzy dead-zone input. IEEE Trans. Syst. Man Cybern. 23(1), 193-204 (2015)

30. Wang, F., Liu, Z., Lai, G.Y.: Fuzzy adaptive control of nonlinear uncertain plants with unknown dead zone output. Fuzzy Sets Syst. 263(1), 27-48 (2015)

31. Liu, Y.J., Tong, S.C.: Adaptive neural network tracking control of uncertain nonlinear discrete-time systems with nonaffine dead-zone input. IEEE Trans. Syst. Man Cybern. 45(3), 497-505 (2015)

32. Liu, Y.J., Tong, S.C.: Adaptive fuzzy control for a class of nonlinear discrete-time systems with backlash. IEEE Trans. Fuzzy Syst. 22(5), 1359-1365 (2014)

33. Zhang, H., Xia, Y.: Adaptive tracking control for a class of stochastic switched systems. Int. J. Control 91(6), 1-16 (2018)

34. Tong, S.C., Li, Y., Li, Y.M., Liu, Y.J.: Observer-based adaptive fuzzy backstepping control for a class of stochastic nonlinear strict-feedback systems. IEEE Trans. Syst. Man Cybern., Part B, Cybern. 41(6), 1693-1704 (2011)

35. Li, J., Chen, W.S., Li, J.M.: Adaptive NN output-feedback decentralized stabilization for a class of large-scale stochastic nonlinear strict-feedback systems. Int. J. Robust Nonlinear Control 21(4), 452-472 (2011)

36. Shen, Y., Han, Y., Shahnazi, R., Haghani, A.: Fuzzy adaptive tracking control of constrained nonlinear switched stochastic pure-feedback systems. IEEE Trans. Cybern. 47(3), 579-588 (2017)

37. Si, W.J., Dong, X.D.: Adaptive neural control for MIMO stochastic nonlinear pure-feedback systems with input saturation and full-state constraints. Neurocomputing 275, 298-307 (2018)

38. Ma, H., Liu, Y., Ye, D.: Adaptive output feedback tracking control for non-linear switched stochastic systems with unknown control directions. IET Control Theory Appl. 12(4), 484-494 (2018)

39. Han, J., Zhang, H., Wang, Y., Zhang, K.: Fault estimation and fault-tolerant control for switched fuzzy stochastic fystems. IEEE Trans. Fuzzy Syst. 99, 1 (2018)

40. Deng, H., Krstic, M.: Output-feedback stochastic nonlinear stabilization. IEEE Trans. Autom. Control 44(2), 328-333 (1999)

41. Wu, Z., Xie, X., Shi, P.: Backstepping controller design for a class of stochastic nonlinear systems with Markovian switching. Automatica 45(4), 997-1004 (2009)

42. Xia, Y., Fu, M., Shi, P.: Adaptive backstepping controller design for stochastic jump systems. IEEE Trans. Autom. Control 54(12), 2853-2859 (2009)

43. Zhao, X., Zheng, X., Niu, B., Liu, L.: Adaptive tracking control for a class of uncertain switched nonlinear systems. Automatica 52, 185-191 (2015)

44. Liu, S.J., Zhang, J.F., Jiang, Z.P.: Decentralized adaptive output-feedback stabilization for large-scale stochastic nonlinear systems. Automatica 43(2), 238-251 (2007)

45. Wang, M., Zhang, S.Y., Chen, B., Luo, F.: Direct adaptive neural control for stabilization of nonlinear time-delay systems. Sci. China Inf. Sci. 53(4), 800-812 (2010)

46. Wang, H.Q., Chen, B., Liu, X.P., Liu, K.F., Lin, C.: Robust adaptive fuzzy tracking control for pure-feedback stochastic nonlinear systems with input constraints. IEEE Trans. Cybern. 43(6), 2093-2104 (2013)

47. Wang, F., Liu, Z., Zhang, Y., Philip Chen, C.L.: Adaptive fuzzy control for a class of stochastic pure-feedback nonlinear systems with unknown hysteresis. IEEE Trans. Fuzzy Syst. 24(1), 140-152 (2016)

48. Yu, Z.X., Li, S.G., Du, H.B.: Razumikhin-Nussbaum-lemma-based adaptive neural control for uncertain stochastic pure-feedback nonlinear systems with time-varying delays. Int. J. Robust Nonlinear Control 23(11), 1214-1239 (2013)

49. Chen, B., Liu, X., Liu, K., Lin, C.: Direct adaptive fuzzy control of nonlinear strict-feedback systems. Automatica 45(6), 1530-1535 (2009)

50. Wang, H.Q., Chen, B., Liu, K.F., Liu, X.P., Lin, C.: Adaptive neural tracking control for a class of non-strict-feedback stochastic nonlinear systems with unknown backlash-like hysteresis. IEEE Trans. Neural Netw. 25(5), 947-958 (2014)

51. Ma, R.C., Zhao, J.: Backstepping design for global stabilization of switched nonlinear systems in lower triangular form under arbitrary switchings. Automatica 46, 1819-1823 (2010) 